\title{
MHD nonaligned stagnation point flow of second grade fluid towards a porous rotating disk
}

https://doi.org/10.1515/nleng-2017-0063

Received January 13, 2017; revised February 9, 2018; accepted February $10,2018$.

\begin{abstract}
This paper provides analytical solution of the non-aligned stagnation point flow of second grade fluid over a porous rotating disk in the presence of a magnetic field and suction/injection at the disk surface. The mathematical formulation of the fluid model is obtained in terms of partial differential equations (PDEs). The PDEs governing the motion are transformed into a system of ordinary differential equations (ODEs) by means of a similarity transformation and these corresponding nonlinear ODEs are solved by employing the homotopy analysis method (HAM) and the convergence analysis of the presented method is also performed graphically. An inclusion of the influences of various admissible parameters has been shown numerically and graphically on the flow field. Furthermore, comparison is made and it concedes that the obtained results are found to be in good agreement with results existing in literature.
\end{abstract}

Keywords: second grade fluid; stagnation point; magnetohydrodynamic; rotating disk

\section{Introduction}

There are a limited number of cases for which the NavierStokes equations have exact solutions. Due to the incompetency of these equations, it has led to the consideration of complex dynamics such as non-Newtonian fluid flow. Recently, the study of non-Newtonian fluid has attained significant importance due to a paramount characteristic that distinguishes the non-Newtonian fluids from other

\footnotetext{
*Corresponding Author: Najeeb Alam Khan, Department of Mathematics, University of Karachi, Karachi 75270, Pakistan, E-mail: njbalam@yahoo.com

Farah Naz, Department of Mathematics, University of Karachi, Karachi 75270, Pakistan

Nadeem Alam Khan, Department of Computer Science, Iqra University, Gulshan Campus Karachi, Pakistan

Saif Ullah, Department of Mathematics, Government College University Lahore, Lahore 54000, Pakistan
}

fluids where the shear stress not only consisting of viscous property but also has an elastic factor, for that reason the non-Newtonian fluid is known as visco-elastic fluid. Non-Newtonian fluids are those fluids which have a wide variety of significant applications specifically in industries. Such applications include ejection of polymer fluids, blood, oils and lotion, food stuff, exotic coatings, drilling fluids. The subclass of non-Newtonian fluids which have simplest model of differential type fluids namely second grade fluid, can be characterized by nonlinear relationship between the shear stress and the shear rate and in which viscosity cannot be constant. A host of various researchers has put forward their ideas towards the flow analysis of second grade fluid by taking the various configurations. Ali et al. [1] studied the non-Newtonian fluid flow, which is passing through the porous channels and the flow considered to be two-dimensional and homotopy perturbation method was used to solve the equations which govern the flow. To inspect the comprehensive study of flow in a channel with varying width, Siddiqui et al. [2] considered the creeping flow of second grade fluid through the medium with variable width and also examined the slip effects on the flow. The analysis of the flow with heat transfer characteristics and associated with boundary conditions has significant importance, therefore, Hayat et al. [3] investigated the second grade fluid flow past through a stretching surface and determined the series solution with an effect of heat transfer properties. Rashidi et al. [4] studied the flow of a second grade fluid passing through a porous medium and analyzed the heat transfer in a fluid.

The flow arising out over a rotating disk has achieved much acceptance and previous literature has dealt many problems involving the flows occurring due to rotating disk. Von Karman [5] was the first, who studied the motion of a viscous fluid over an infinite rotating disk and it assumes that the disk is at rest. Apart from that, fluid flow associated with a rotating disk has massive applications and many researchers have provided these types of flows to analyze the scientific insights. The shrinking flow of an incompressible second grade fluid occurred between two porous walls has been analyzed by Hayat et al. [6]. The incompressible fluid flow occurring between two co-rotating disks has been carried out by Batista [7], who provided 
the solution of the derived ordinary differential equations by evaluating the unknown functions. Turkyilmazoglu [8] paid his attention to determine the flow characteristics of nanofluid over a rotating disk and observed the heat transfer effect on the flow.

Nevertheless, there may be some cases where the rotating disk and stagnation flow axes are not to be aligned and stagnation flow is pointedly be non-aligned. These types of flows offer non-aligned flows where both the axes of rotation and stagnation are parallel, but said to be noncoinciding axes. Many authors have been fascinated by considering non-aligned rotating disk flows and assessed these types of flows with different effects. The pioneering study about an off-centered stagnation flow of viscous fluid over a rotating disk has been presented by Wang [9]. The suitable similarity transformation has also been implemented by Wang [9] for converting into a meaningful system for which the solution is existed. Further, Dinarvand [10] considered the problem of Wang [9] and gave an analytic solution via the homotopy analytical technique. Erfani et al. [11] analyzed the steady flow over a rotating disk which is off-centered and examined the solution of flow by utilization of modified differential transform method, where Nourbakhsh et al. [12] introduced two auxiliary parameters in homotopy analysis method and obtained the solution of off-centered stagnation flow over a rotating disk.

The several scientists and researchers have paid a significant portion of their attention to analyze the flow occurring due to a rotating disk for non-Newtonian fluid subjected to uniform magnetic field. They consumed great efforts to examine the solutions for the equations which govern the flow over a rotating disk. In 1970, Alfven [13] was the first person who used the term of magnetohydrodynamic (MHD). Later, Turkyilmazoglu [14] analyzed the three dimensional stagnation point flow of an electrically conducting fluid occurring as a result of stretchable rotating disk and also taking magnetic field effect into account. The effect of an external uniform magnetic field on the fluid flow over a disk surface with heat transfer characteristics was determined in $[15,16]$.

A great extent of research has been concerned to consider the flow with the effect of suction or injection velocity and it developed various features of the problem which have been studied by several investigators. The solution of boundary layer flow of steady viscous fluid due to rotating disk with uniform suction or injection has been proposed by Turkyilmazoglu [17]. The study which deals with the consideration of an incompressible non-Newtonian fluid flow passing through a permeable medium has been presented by Attia [18].
The significant objective of the present study is to organize the problem concerning with flow characteristics of second grade fluid over a rotating disk. The similarity transformation is utilized for converting the three dimensional partial differential equations into ordinary differential equations. The series solution of considered problem is obtained through the application of the homotopy analysis method. The solutions that have been attained satisfy the differential equation and all imposed boundary conditions as well. The convergence criteria for the series solution have also been considered. Moreover, at the end of this paper, the effects of emerging flow parameters are shown on velocity profiles and discussed in detail. The comparison is also made with the previous results published in ref. [9].

\section{Analysis and mathematical formulation of the problem}

Consider the second grade fluid flow near the stagnation point occurring because of a non-aligned infinite rotating porous disk. The disk is rotating with an angular velocity $\Omega$ and its flow is about an axis which is along with $z$ axis and has a distance $b$ from the center of the disk. Let $[u, v, w]$ are velocity constituents along their respective $x, y$ and $z$ directions. Moreover, it is assumed that an external uniform magnetic field of strength $B_{0}$ is applied in the $z$-direction that produces magnetic effect in the $x$ and $y$ directions. Therefore, the magnetic field is $B=\left(0,0, B_{0}\right)$. The magnetic Reynolds number is considered to be very small, therefore the induced magnetic field is negligible. At the disk surface, a uniform injection or suction is also applied for the entire range from large injection velocities to large suction velocities. The geometrical configuration of the physical model has been presented in Fig. 1.

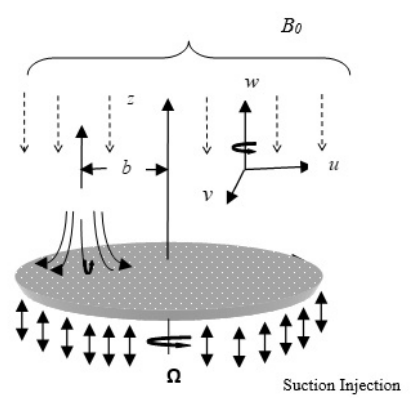

Fig. 1: Flow structure of physical model 
The fundamental equations for a second grade fluid [19-21] can be given as:

$$
\begin{gathered}
\operatorname{div} V=0 \\
\rho \frac{d V}{d t}=-\operatorname{grad} p+\operatorname{div} S+\rho f \\
S=\mu\left(\operatorname{grad} V+(\operatorname{grad} V)^{T}\right)+\alpha_{1}\left(\frac{d A_{1}}{d t}+A_{1}(\operatorname{grad} V)+(\operatorname{grad} V)^{T} A_{1}\right)+\alpha_{2}\left(\operatorname{grad} V+(\operatorname{grad} V)^{T}\right)^{2} \\
\rho f=\sigma(V \times \mathbf{B}) \times \mathbf{B}
\end{gathered}
$$

where $\mu$ is the dynamic viscosity coefficient, normal stress moduli are $\alpha_{1}$ and $\alpha_{2}$ with the conditions fulfill by these material parameters $\mu \geq 0, \alpha_{1} \geq 0, \alpha_{1}+\alpha_{2}=0$. In above, $A_{1}=\operatorname{grad} V+(\operatorname{grad} V)^{T}$ is kinematical tensor which known as Rivilin-Ericksen tensor, $\frac{d}{d t}$ gives material time derivative, $p, \rho f$ and $S$ represent the hydrostatic pressure, MHD body force or Lorentz force and extra stress tensor, respectively. By recalling the rules of vectors multiplication, the Lorentz force can also be written as:

$$
\rho f=-\sigma[V(\mathbf{B} . \mathbf{B})-\mathbf{B}(B . V)]
$$

Therefore, the continuity and momentum equations which govern the flow are given as:

$$
\left.\begin{array}{c}
u x+v_{y}+w z=0 \\
u \frac{\partial u}{\partial x}+v \frac{\partial u}{\partial y}+w \frac{\partial u}{\partial z}=-\frac{1}{\rho} \frac{\partial p}{\partial x}+v\left(\frac{\partial^{2} u}{\partial x^{2}}+\frac{\partial^{2} u}{\partial y^{2}}+\frac{\partial^{2} u}{\partial z^{2}}\right) \\
+\frac{\alpha_{1}}{\rho}\left[\begin{array}{l}
\left(u \frac{\partial}{\partial x}+v \frac{\partial}{\partial y}+w \frac{\partial}{\partial z}\right)\left(\frac{\partial^{2} u}{\partial x^{2}}+\frac{\partial^{2} u}{\partial y^{2}}+\frac{\partial^{2} u}{\partial z^{2}}\right)+9 \frac{\partial u}{\partial x} \frac{\partial^{2} u}{\partial x^{2}}+4 \frac{\partial v}{\partial x} \frac{\partial^{2} u}{\partial x \partial y}+2 \frac{\partial w}{\partial x} \frac{\partial^{2} u}{\partial z \partial x} \\
+4 \frac{\partial u}{\partial y} \frac{\partial^{2} u}{\partial x \partial y}+2 \frac{\partial v}{\partial y} \frac{\partial^{2} u}{\partial y^{2}}+\frac{\partial w}{\partial y} \frac{\partial^{2} u}{\partial y \partial z}+3 \frac{\partial u}{\partial y} \frac{\partial^{2} v}{\partial x^{2}}+4 \frac{\partial v}{\partial y} \frac{\partial^{2} v}{\partial x \partial y}+2 \frac{\partial w}{\partial y} \frac{\partial^{2} v}{\partial x \partial z}+4 \frac{\partial u}{\partial z} \frac{\partial^{2} u}{\partial z \partial x} \\
+2 \frac{\partial v}{\partial y \partial z}+\frac{\partial w}{\partial z} \frac{\partial^{2} u}{\partial z^{2}}+3 \frac{\partial u}{\partial z} \frac{\partial^{2} w}{\partial x^{2}}+2 \frac{\partial v}{\partial z} \frac{\partial^{2} w}{\partial x \partial y}+4 \frac{\partial w}{\partial z} \frac{\partial^{2} w}{\partial x \partial z}+4 \frac{\partial v}{\partial x} \frac{\partial^{2} v}{\partial x^{2}}+4 \frac{\partial w}{\partial x} \frac{\partial^{2} w}{\partial x^{2}} \\
+3 \frac{\partial u}{\partial x} \frac{\partial^{2} u}{\partial y^{2}}+\frac{\partial u}{\partial y} \frac{\partial^{2} v}{\partial y^{2}}+2 \frac{\partial v}{\partial x} \frac{\partial^{2} v}{\partial y^{2}}+\left(\frac{\partial u}{\partial z}+\frac{\partial w}{\partial x}\right)\left(\frac{\partial v}{\partial y} \frac{\partial v}{\partial y}+v \frac{\partial^{2} v}{\partial y^{2}}\right)+v \frac{\partial v}{\partial y}\left(\frac{\partial^{2} u}{\partial z \partial y}+\frac{\partial^{2} w}{\partial x \partial y}\right) \\
+\frac{\partial w}{\partial y} \frac{\partial^{2} w}{\partial x \partial y}+\frac{\partial w}{\partial x} \frac{\partial^{2} w}{\partial y^{2}}+2 \frac{\partial w}{\partial x} \frac{\partial^{2} w}{\partial z^{2}}+3 \frac{\partial u}{\partial x} \frac{\partial^{2} u}{\partial z^{2}}+\frac{\partial u}{\partial y} \frac{\partial^{2} v}{\partial z^{2}}+2 \frac{\partial v}{\partial x} \frac{\partial^{2} v}{\partial z^{2}}+2 \frac{\partial v}{\partial z} \frac{\partial^{2} v}{\partial x \partial z}+\frac{\partial u}{\partial z} \frac{\partial^{2} w}{\partial z^{2}} \\
+\frac{\partial u}{\partial x} \frac{\partial^{2} u}{\partial x^{2}}+4 \frac{\partial v}{\partial x} \frac{\partial^{2} u}{\partial x \partial y}+4 \frac{\partial w}{\partial x} \frac{\partial^{2} u}{\partial z \partial x}-2 \frac{\partial u}{\partial x} \frac{\partial^{2} w}{\partial x \partial z}-2 \frac{\partial u}{\partial x} \frac{\partial^{2} v}{\partial x \partial y}+2 \frac{\partial u}{\partial y} \frac{\partial^{2} u}{\partial x \partial y}-2 \frac{\partial u}{\partial y} \frac{\partial^{2} w}{\partial y \partial z} \\
+2 \frac{\partial v}{\partial y} \frac{\partial^{2} u}{\partial y^{2}}+\frac{\partial w}{\partial y} \frac{\partial^{2} u}{\partial y \partial z}+2 \frac{\partial u}{\partial y} \frac{\partial^{2} v}{\partial x^{2}}+2 \frac{\partial v}{\partial y} \frac{\partial^{2} v}{\partial x \partial y}+\frac{\partial u}{\partial z} \frac{\partial^{2} u}{\partial x \partial z}-\frac{\partial u}{\partial z} \frac{\partial^{2} w}{\partial z^{2}}-2 \frac{\partial u}{\partial z} \frac{\partial^{2} v}{\partial y \partial z} \\
+\frac{\partial v}{\partial z} \frac{\partial^{2} u}{\partial y \partial z}+2 \frac{\partial w}{\partial z} \frac{\partial^{2} u}{\partial z^{2}}+2 \frac{\partial u}{\partial z} \frac{\partial^{2} w}{\partial x^{2}}+\frac{\partial v}{\partial z} \frac{\partial^{2} w}{\partial x \partial y}+2 \frac{\partial w}{\partial z} \frac{\partial^{2} w}{\partial x \partial z}+2 \frac{\partial v}{\partial x} \frac{\partial^{2} v}{\partial x^{2}}+2 \frac{\partial w}{\partial x} \frac{\partial^{2} w}{\partial x^{2}} \\
+2 \frac{\partial u}{\partial x} \frac{\partial^{2} u}{\partial y^{2}}+2 \frac{\partial v}{\partial x} \frac{\partial^{2} v}{\partial y^{2}}+\frac{\partial u}{\partial z} \frac{\partial^{2} w}{\partial y^{2}}+\frac{\partial w}{\partial x} \frac{\partial^{2} w}{\partial y^{2}}+\frac{\partial w}{\partial x} \frac{\partial^{2} v}{\partial z \partial y}+\frac{\partial w}{\partial y} \frac{\partial^{2} w}{\partial x \partial y}+2 \frac{\partial u}{\partial x} \frac{\partial^{2} u}{\partial z^{2}} \\
+\frac{\partial^{2} u}{\partial y \partial z}+\frac{\partial^{2} v}{\partial x \partial z}+\frac{\partial^{2} v}{\partial z^{2}}+\frac{\partial^{2} w}{\partial y \partial z}+2 \frac{\partial w}{\partial x} \frac{\partial^{2} w}{\partial z^{2}}
\end{array}\right]-\frac{\sigma B_{0}^{2} u}{\rho}
\end{array}\right]
$$




$$
\begin{aligned}
& u \frac{\partial v}{\partial x}+v \frac{\partial v}{\partial y}+w \frac{\partial v}{\partial z}=-\frac{1}{\rho} \frac{\partial p}{\partial y}+v\left(\frac{\partial^{2} v}{\partial x^{2}}+\frac{\partial^{2} v}{\partial y^{2}}+\frac{\partial^{2} v}{\partial z^{2}}\right) \\
& +\frac{\alpha_{1}}{\rho}\left[\begin{array}{l}
\left(u \frac{\partial}{\partial x}+v \frac{\partial}{\partial y}+w \frac{\partial}{\partial z}\right)\left(\frac{\partial^{2} v}{\partial x^{2}}+\frac{\partial^{2} v}{\partial y^{2}}+\frac{\partial^{2} v}{\partial z^{2}}\right)+4 \frac{\partial u}{\partial x} \frac{\partial^{2} u}{\partial y \partial x}+3 \frac{\partial v}{\partial x} \frac{\partial^{2} u}{\partial y^{2}}+2 \frac{\partial w}{\partial x} \frac{\partial^{2} u}{\partial y \partial z} \\
+2 \frac{\partial u}{\partial x} \frac{\partial^{2} v}{\partial x^{2}}+3 \frac{\partial v}{\partial x} \frac{\partial^{2} v}{\partial x \partial y}+2 \frac{\partial w}{\partial x} \frac{\partial^{2} v}{\partial x \partial z}+\frac{\partial u}{\partial y} \frac{\partial^{2} v}{\partial x \partial y}+\frac{\partial w}{\partial y} \frac{\partial^{2} v}{\partial y \partial z}+2 \frac{\partial u}{\partial z} \frac{\partial^{2} v}{\partial x \partial z}+3 \frac{\partial v}{\partial z} \frac{\partial^{2} v}{\partial y \partial z} \\
+2 \frac{\partial w}{\partial z} \frac{\partial^{2} v}{\partial z^{2}}+2 \frac{\partial u}{\partial z} \frac{\partial^{2} w}{\partial x \partial y}+3 \frac{\partial v}{\partial z} \frac{\partial^{2} w}{\partial y^{2}}+4 \frac{\partial w}{\partial z} \frac{\partial^{2} w}{\partial y \partial z}-\frac{\partial v}{\partial x} \frac{\partial^{2} w}{\partial x \partial z}-3 \frac{\partial u}{\partial y} \frac{\partial^{2} w}{\partial x \partial z}+3 \frac{\partial^{2} w}{\partial y}+2 \frac{\partial^{2} v}{\partial x^{2}} \\
+4 \frac{\partial w}{\partial y} \frac{\partial^{2} w}{\partial x \partial y}-8 \frac{\partial^{2}}{\partial y} \frac{\partial^{2}}{\partial y^{2}}+\frac{\partial v}{\partial x} \frac{\partial^{2} v}{\partial z^{2}}+2 \frac{\partial u}{\partial y} \frac{\partial^{2} w}{\partial y \partial z}-\frac{\partial w}{\partial y} \frac{\partial^{2} w}{\partial z^{2}}+4 \frac{\partial u}{\partial y} \frac{\partial^{2} u}{\partial y^{2}} \\
-2 \frac{\partial u}{\partial y} \frac{\partial^{2} u}{\partial x^{2}}-\frac{\partial v}{\partial y} \frac{\partial^{2} u}{\partial x \partial y} \frac{\partial^{2} u}{\partial y \partial z}+3 \frac{\partial v}{\partial y} \frac{\partial^{2} v}{\partial z^{2}}-\frac{\partial v}{\partial z} \frac{\partial^{2} u}{\partial x \partial z}-3 \frac{\partial w}{\partial y} \frac{\partial^{2} u}{\partial x \partial z}
\end{array}\right] \\
& +\frac{\alpha_{2}}{\rho}\left[\begin{array}{c}
2 \frac{\partial u}{\partial x} \frac{\partial^{2} u}{\partial y \partial x}+2 \frac{\partial v}{\partial x} \frac{\partial^{2} u}{\partial y^{2}}+\frac{\partial w}{\partial x} \frac{\partial^{2} u}{\partial y \partial z}+2 \frac{\partial u}{\partial x} \frac{\partial^{2} v}{\partial x^{2}}+3 \frac{\partial v}{\partial x} \frac{\partial^{2} v}{\partial x \partial y}+2 \frac{\partial w}{\partial x} \frac{\partial^{2} v}{\partial x \partial z}+\frac{\partial u}{\partial y} \frac{\partial^{2} v}{\partial x \partial y} \\
-2 \frac{\partial u}{\partial y} \frac{\partial^{2} w}{\partial x \partial z}+\frac{\partial w}{\partial y} \frac{\partial^{2} v}{\partial y \partial z}-2 \frac{\partial w}{\partial y} \frac{\partial^{2} u}{\partial x \partial z}-\frac{\partial w}{\partial y} \frac{\partial^{2} w}{\partial z^{2}}+2 \frac{\partial u}{\partial z} \frac{\partial^{2} v}{\partial x \partial z}+2 \frac{\partial v}{\partial z} \frac{\partial^{2} v}{\partial y \partial z}-\frac{\partial v}{\partial z} \frac{\partial^{2} w}{\partial z^{2}} \\
+\frac{\partial}{\partial z \partial x}+2 \frac{\partial w}{\partial z} \frac{\partial^{2} v}{\partial z^{2}}+\frac{\partial u}{\partial z} \frac{\partial^{2} w}{\partial x \partial y}+2 \frac{\partial v}{\partial z} \frac{\partial^{2} w}{\partial y^{2}}+2 \frac{\partial w}{\partial z} \frac{\partial^{2} w}{\partial y \partial z}+\frac{\partial v}{\partial x} \frac{\partial^{2} u}{\partial x^{2}}+2 \frac{\partial v}{\partial y} \frac{\partial^{2} v}{\partial x^{2}} \\
+\frac{\partial w}{\partial x} \frac{\partial^{2} w}{\partial x \partial y}+\frac{\partial v}{\partial z} \frac{\partial^{2} w}{\partial x^{2}}+\frac{\partial w}{\partial y} \frac{\partial^{2} w}{\partial x^{2}}+2 \frac{\partial u}{\partial y} \frac{\partial^{2} u}{\partial y^{2}}+2 \frac{\partial w}{\partial y} \frac{\partial^{2} w}{\partial y^{2}}+6 \frac{\partial v}{\partial y} \frac{\partial^{2} v}{\partial y^{2}}+\frac{\partial v}{\partial x} \frac{\partial^{2} u}{\partial z^{2}} \\
+\frac{\partial u}{\partial y} \frac{\partial^{2} u}{\partial z^{2}}-\frac{\partial u}{\partial y} \frac{\partial^{2} u}{\partial x^{2}}+\frac{\partial u}{\partial z} \frac{\partial^{2} u}{\partial y \partial z}+2 \frac{\partial v}{\partial y} \frac{\partial^{2} v}{\partial z^{2}}
\end{array}\right]-\frac{\sigma B_{0}^{2} v}{\rho}
\end{aligned}
$$




$$
\begin{aligned}
& u \frac{\partial w}{\partial x}+v \frac{\partial w}{\partial y}+w \frac{\partial w}{\partial z}=-\frac{1}{\rho} \frac{\partial p}{\partial z}+v\left(\frac{\partial^{2} w}{\partial x^{2}}+\frac{\partial^{2} w}{\partial y^{2}}+\frac{\partial^{2} w}{\partial z^{2}}\right) \\
& +\frac{\alpha_{1}}{\rho}\left[\begin{array}{l}
\left(u \frac{\partial}{\partial x}+v \frac{\partial}{\partial y}+w \frac{\partial}{\partial z}\right)\left(\frac{\partial^{2} w}{\partial x^{2}}+\frac{\partial^{2} w}{\partial y^{2}}+\frac{\partial^{2} w}{\partial z^{2}}\right)+4 \frac{\partial u}{\partial x} \frac{\partial^{2} u}{\partial x \partial z}+2 \frac{\partial v}{\partial x} \frac{\partial^{2} u}{\partial y \partial z}+3 \frac{\partial w}{\partial x} \frac{\partial^{2} u}{\partial z^{2}} \\
+2 \frac{\partial u}{\partial x} \frac{\partial^{2} w}{\partial x^{2}}+2 \frac{\partial v}{\partial x} \frac{\partial^{2} w}{\partial x \partial y}+4 \frac{\partial w}{\partial x} \frac{\partial^{2} w}{\partial x \partial z}+2 \frac{\partial u}{\partial y} \frac{\partial^{2} w}{\partial x \partial y}+2 \frac{\partial v}{\partial y} \frac{\partial^{2} w}{\partial y^{2}}+4 \frac{\partial w}{\partial y} \frac{\partial^{2} w}{\partial y \partial z}+\frac{\partial^{2} u}{\partial x^{2}} \frac{\partial w}{\partial x} \\
+5 \frac{\partial u}{\partial z} \frac{\partial^{2} w}{\partial x \partial z}+\frac{\partial w}{\partial z} \frac{\partial^{2} u}{\partial z \partial x}+4 \frac{\partial^{2} v}{\partial y} \frac{\partial w}{\partial y}+2 \frac{\partial^{2} v}{\partial z \partial y}+3 \frac{\partial^{2} v}{\partial x^{2}}+2 \frac{\partial v}{\partial x} \frac{\partial^{2} v}{\partial z \partial x}+3 \frac{\partial w}{\partial z} \frac{\partial^{2} w}{\partial x^{2}}+2 \frac{\partial u}{\partial y} \frac{\partial^{2} v}{\partial z \partial x}+\frac{\partial v}{\partial z} \frac{\partial^{2} u}{\partial y \partial x} \frac{\partial^{2} u}{\partial y^{2}}+2 \frac{\partial u}{\partial z} \frac{\partial^{2} u}{\partial y^{2}}+2 \frac{\partial u}{\partial y} \frac{\partial^{2} u}{\partial z \partial y} \\
+\frac{\partial w}{\partial y} \frac{\partial^{2} v}{\partial y^{2}}+3 \frac{\partial v}{\partial z} \frac{\partial^{2} v}{\partial y^{2}}+3 \frac{\partial w}{\partial z} \frac{\partial^{2} w}{\partial y^{2}}+\frac{\partial u}{\partial z} \frac{\partial^{2} v}{\partial x \partial y}+\frac{\partial w}{\partial z} \frac{\partial^{2} v}{\partial z \partial y}+10 \frac{\partial w}{\partial z} \frac{\partial^{2} w}{\partial z^{2}}+4 \frac{\partial u}{\partial z} \frac{\partial^{2} u}{\partial z^{2}} \\
+4 \frac{\partial v}{\partial z} \frac{\partial^{2} v}{\partial z^{2}}
\end{array}\right] \\
& +\frac{\alpha_{2}}{\rho}\left[\begin{array}{l}
2 \frac{\partial u}{\partial x} \frac{\partial^{2} u}{\partial x \partial z}+\frac{\partial v}{\partial x} \frac{\partial^{2} u}{\partial z \partial y}+2 \frac{\partial w}{\partial x} \frac{\partial^{2} u}{\partial z^{2}}+2 \frac{\partial u}{\partial x} \frac{\partial^{2} w}{\partial x^{2}}+2 \frac{\partial v}{\partial x} \frac{\partial^{2} w}{\partial x \partial y}+\frac{\partial w}{\partial x} \frac{\partial^{2} w}{\partial x \partial z}-2 \frac{\partial w}{\partial x} \frac{\partial^{2} v}{\partial x \partial y} \\
-\frac{\partial w}{\partial x} \frac{\partial^{2} u}{\partial x^{2}}+2 \frac{\partial u}{\partial y} \frac{\partial^{2} w}{\partial x \partial y}+2 \frac{\partial v}{\partial y} \frac{\partial^{2} w}{\partial y^{2}}+\frac{\partial w}{\partial y} \frac{\partial^{2} w}{\partial y \partial z}-\frac{\partial w}{\partial y} \frac{\partial^{2} v}{\partial y^{2}}-2 \frac{\partial w}{\partial y} \frac{\partial^{2} u}{\partial x \partial y}+\frac{\partial v}{\partial z} \frac{\partial^{2} w}{\partial y \partial z} \\
+-2 \frac{\partial v}{\partial z} \frac{\partial^{2} u}{\partial x \partial y}+\frac{\partial v}{\partial x} \frac{\partial^{2} v}{\partial z \partial x}+\frac{\partial u}{\partial y} \frac{\partial^{2} v}{\partial z \partial x}+\frac{\partial w}{\partial y} \frac{\partial^{2} v}{\partial x^{2}}+\frac{\partial v}{\partial z}-\frac{\partial u}{\partial x^{2}}+\frac{\partial^{2}}{\partial z} \frac{\partial^{2} u}{\partial x^{2}}+2 \frac{\partial w}{\partial z} \frac{\partial^{2} w}{\partial x \partial} \\
+\frac{\partial w}{\partial x} \frac{\partial^{2} u}{\partial y^{2}}+\frac{\partial u}{\partial z} \frac{\partial^{2} u}{\partial y^{2}}+2 \frac{\partial v}{\partial y} \frac{\partial^{2} v}{\partial z \partial y}+2 \frac{\partial^{2} u}{\partial x \partial z}-8 \frac{\partial w}{\partial z} \frac{\partial^{2} v}{\partial y \partial z}-2 \frac{\partial^{2} w}{\partial y^{2}}+2 \frac{\partial u}{\partial z} \frac{\partial^{2} u}{\partial z^{2}}+2 \frac{\partial v}{\partial z} \frac{\partial^{2} v}{\partial z^{2}}+2 \frac{\partial w}{\partial y} \frac{\partial^{2} v}{\partial z^{2}} \frac{\partial^{2} u}{\partial z \partial y}
\end{array}\right]
\end{aligned}
$$

The boundary conditions satisfying the fluid model can be given as:

$$
\begin{gathered}
(u, v, w)=\left(-\Omega y, \Omega(x-b), w_{w}\right), \text { at } \eta \rightarrow 0 \\
(u, v, w)=(a x, a y,-2 a z), \text { at } \eta \rightarrow \infty
\end{gathered}
$$

where $a$ and $v$ represent the strength of the stagnation flow and kinematic viscosity. $w_{w}<0$ corresponds to suction and $w_{w}>0$ corresponds to injection.

\section{Reduction to ordinary differential equations}

The similarity transformation provided by Wang [9] is defined as:

$$
\begin{gathered}
u=a x f^{\prime}(\eta)-\Omega y g(\eta)+b \Omega k(\eta), \\
v=a y f^{\prime}(\eta)+\Omega x g(\eta)+b \Omega h(\eta), \\
w=-2 \sqrt{a v} f(\eta), \eta=z \sqrt{a / v}
\end{gathered}
$$

where $\left(\star^{\prime}\right)=\frac{\partial\left(^{\star}\right)}{\partial \eta}$, by utilizing the above similarity transformations in Eqs. (6)-(9), the equation of continuity is satisfied, however, the leading partial differential equations are transformed to the form specified by:

$$
\begin{gathered}
f^{\prime \prime \prime}-M f^{\prime}+2 f f^{\prime \prime}+\alpha^{2} g^{2}-f^{2}+1+W e\left(2 f^{\prime \prime 2}-2 f f^{\prime \prime \prime \prime}+\lambda\left(-\alpha^{2} g^{2}+f^{\prime \prime 2}-2 f^{\prime} f^{\prime \prime \prime}\right)\right)=0 \\
g^{\prime \prime}-M g-2 g f^{\prime}+2 g^{\prime} f+W e\left(2 g^{\prime} f^{\prime \prime}-2 f g^{\prime \prime \prime}+\lambda\left(2 g^{\prime} f^{\prime \prime}-2 f^{\prime} g^{\prime \prime}\right)\right)=0
\end{gathered}
$$




$$
\begin{gathered}
k^{\prime \prime}-M \mathrm{k}+\alpha \mathrm{gh}-\mathrm{kf}^{\prime}+2 \mathrm{fk}^{\prime}+\mathrm{We}\left(2 \mathrm{k}^{\prime} \mathrm{f}^{\prime \prime}-\alpha \mathrm{hg}^{\prime \prime}+\alpha \mathrm{gh}^{\prime \prime}-\mathrm{f}^{\prime} \mathrm{k}^{\prime \prime}+\mathrm{kf}^{\prime \prime \prime}-2 \mathrm{fk}^{\prime \prime \prime}+\lambda\left(\mathrm{k}^{\prime} \mathrm{f}^{\prime \prime}-\alpha \mathrm{g}^{\prime} \mathrm{h}^{\prime}-2 \mathrm{f}^{\prime} \mathrm{k}^{\prime \prime}\right)\right)=0, \\
h^{\prime \prime}-M h-\alpha g k-h f^{\prime}+2 f h^{\prime}+W e\left(\begin{array}{c}
2 h^{\prime} f^{\prime \prime}+\alpha k g^{\prime \prime}-f^{\prime} h^{\prime \prime}-\alpha g k^{\prime \prime} \\
+h f^{\prime \prime \prime}-2 f h^{\prime \prime \prime}+\lambda\left(h^{\prime} f^{\prime \prime}+\alpha g^{\prime} k^{\prime}-2 h^{\prime \prime} f^{\prime}\right)
\end{array}\right)=0
\end{gathered}
$$

and the boundary conditions transformed to:

$$
\begin{gathered}
\left(f(0)-f_{w}, f^{\prime}(0), g(0)-1, k(0), h(0)-1\right)=(0,0,0,0,0) \\
\left(f^{\prime}(\infty)-1, g(\infty), k(\infty), h(\infty)\right)=(0,0,0,0)
\end{gathered}
$$

where $\Omega=a \alpha$ is the non-dimensional rotational parameter, $\lambda=\frac{\alpha_{2}}{\alpha_{1}}$ is the ratio of normal stress moduli, We $=\frac{a \alpha_{1}}{\mu}$ is the Weissenberg number, non-dimensional velocity $f_{w}=\frac{w_{w}}{-2 \sqrt{a v}}, M=\frac{\sigma B_{0}^{2}}{\rho a}$ is the magnetic parameter and where $f_{w}<0$ represents injection while $f_{w}>0$ represents suction.

The pressure $p$ can be obtained by:

$$
p=p_{0}-\rho\left(\frac{a^{2}\left(x^{2}+y^{2}\right)}{2}-\frac{w^{2}}{2}\right)+2 \mu w_{z}+\alpha_{1}\left(4 w_{z}^{2}+2 w w_{z, z}\right)+4 \alpha_{2} w_{z}^{2}
$$

Now, it is transformed to

$$
=p_{0}-\rho\left(\frac{a^{2}\left(x^{2}+y^{2}\right)}{2}\right)+2 a \mu f^{2}(\eta)-4 a \mu f^{\prime}(\eta)+8 a^{2} \alpha_{1} f(\eta) f^{\prime \prime}(\eta)
$$

where $p_{0}$ an $\rho$ represent the pressure at the plate and fluid density and $\left({ }^{\star}\right)_{z}$ gives differentiation with respect to $z$. The shear stress at disk surface for the second grade fluid is given by:

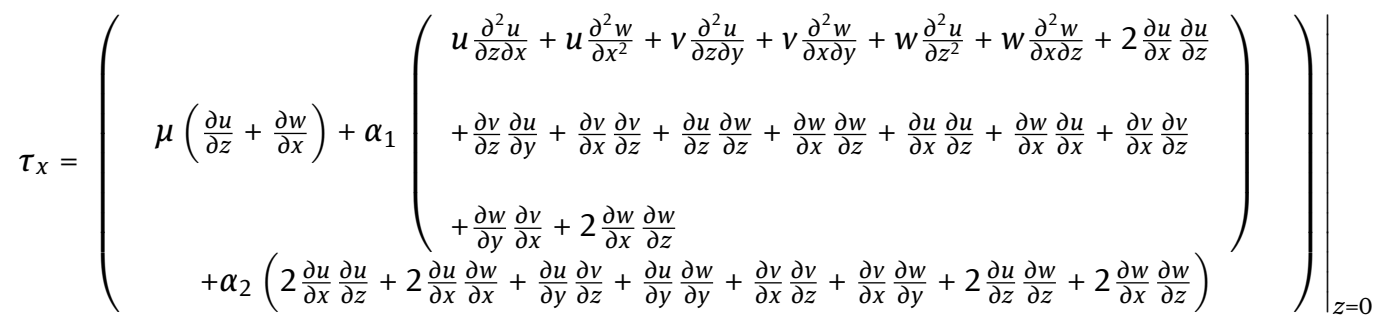

$$
\begin{aligned}
& \tau_{x}=\rho a \sqrt{v a}\left(\begin{array}{c}
x\left(f^{\prime \prime}(0)+W e\left(2 f^{\prime}(0) f^{\prime \prime}(0)-2 \lambda f^{\prime}(0) f^{\prime \prime \prime}(0)-2 f(0) f^{\prime \prime \prime}(0)\right)\right) \\
-\alpha y\left(g^{\prime}(0)+W e\left(2 f^{\prime}(0) g^{\prime}(0)-2 \lambda f^{\prime}(0) g^{\prime}(0)-2 f(0) g^{\prime \prime}(0)\right)\right) \\
-b \alpha\left(\begin{array}{l}
\alpha h(0) g^{\prime}(0)-\alpha g(0) h^{\prime}(0)-f^{\prime}(0) k^{\prime}(0)+2 \lambda f^{\prime}(0) k^{\prime}(0) \\
-k(0) f^{\prime \prime}(0)+2 f(0) k^{\prime \prime}(0)
\end{array}\right)-k^{\prime}(0)
\end{array}\right)
\end{aligned}
$$

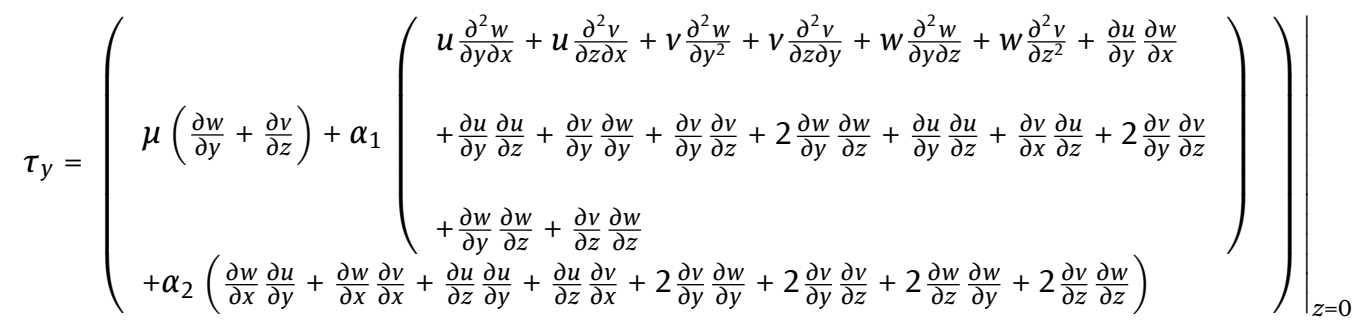

$$
\begin{aligned}
& =\rho a \sqrt{v a}\left[\begin{array}{l}
\alpha x\left(g^{\prime}(0)+W e\left(2 f^{\prime}(0) g^{\prime}(0)-2 \lambda f^{\prime}(0) g^{\prime}(0)-2 f(0) g^{\prime \prime}(0)\right)\right) \\
+y\left(f^{\prime \prime}(0)+W e\left(2 f^{\prime}(0) f^{\prime \prime}(0)-2 \lambda f^{\prime}(0) f^{\prime \prime}(0)-2 f(0) f^{\prime \prime \prime}(0)\right)\right) \\
+b \alpha\left(h^{\prime}(0)+W e\left(\begin{array}{l}
\alpha k(0) g^{\prime}(0)+f^{\prime}(0) h^{\prime}(0)-2 \lambda f^{\prime}(0) h^{\prime}(0)-\alpha g(0) k^{\prime}(0) \\
+h(0) f^{\prime \prime}(0)-2 f(0) h^{\prime \prime}(0)
\end{array}\right)\right)
\end{array}\right]
\end{aligned}
$$


The shear center at the disk surface has been achieved by equating Eqs. (22) and (24) to zero and solving for $(x, y)$. The shear stress is zero at the center of the disk. The torque experienced by the disk of radius is given by:

$$
\tau=\int_{0}^{R} \int_{0}^{2 \pi}\left(\tau_{y} \cos \theta-\tau_{x} \sin \theta\right) r^{2} d \theta d r,
$$

where $(r, \theta)$ are cylindrical coordinates. Since $x=r \cos \theta-b$ and $y=r \sin \theta$, the torque can be found as:

$$
\tau=\frac{\pi}{2} a \sqrt{a v} \alpha R^{4} \rho\left(g^{\prime}(0)-W e\left(2 \lambda f^{\prime}(0) g^{\prime}(0)-2 f^{\prime}(0) g^{\prime}(0)+2 f(0) g^{\prime \prime}(0)\right)\right)
$$

which remain unaltered by the non-alignment in disk and flow axis.

Also, the significant system of second grade fluid presented by Eqs. (13)-(16) can be converted to the Navier-Stokes equations of stagnation flow [9] by setting the values of $M$, We and $\lambda$ to zero.

\section{Analytical approximations by means of HAM}

Homotopy is a fundamental concept in topology, which has been employed in homotopy analysis method (HAM). HAM provides latest development of analytic solutions for highly nonlinear problems. Unlike perturbation methods, it also provides a great degree of freedom with small/large physical parameters.

The auxiliary linear operators $L_{1}[f]$ and $L_{2}[g, k, h]$ have been taken for the Eqs. (13)-(16) as:

$$
\left(L_{1}[f], L_{2}[g, k, h]\right)=\left(\frac{d^{3}}{d \eta^{3}}-\frac{d}{d \eta}, \frac{d^{2}}{d \eta^{2}}-1\right),
$$

Also, the approximations which satisfy the initial conditions in Eqs. (17)-(18) have initial guesses as:

$$
\left(f_{0}(\eta), g_{0}(\eta), k_{0}(\eta), h_{0}(\eta)\right)=\left(f_{w}+\eta-1+e^{-\eta}, e^{-\eta}, \eta e^{-\eta}, e^{-\eta}\right),
$$

satisfying the following properties

$$
L_{1}\left[c_{1}+c_{2} e^{\eta}+c_{3} e^{-\eta}\right]=0, L_{2}\left[c_{4} e^{\eta}+c_{5} e^{-\eta}\right]=0
$$

where $c_{1}, c_{2}, \ldots, c_{5}$ are arbitrary constants.

The zero ${ }^{\text {th }}$ order deformation equations can be constructed as:

$$
\begin{gathered}
L_{1}\left[\breve{f}(\eta, q)-f_{0}(\eta)\right]-q\left[L_{1}\left[\breve{f}(\eta, q)-f_{0}(\eta)\right]+\hbar_{1} N_{1}[\breve{f}(\eta, q), \breve{g}(\eta, q)]\right]=0 \\
L_{2}\left[\breve{g}(\eta, q)-g_{0}(\eta)\right]-q\left[L_{2}\left[\breve{g}(\eta, q)-g_{0}(\eta)\right]+\hbar_{2} N_{2}[\breve{f}(\eta, q), \breve{g}(\eta, q)]\right]=0 \\
L_{2}\left[\breve{k}(\eta, q)-k_{0}(\eta)\right]-q\left[L_{2}\left[\breve{k}(\eta, q)-k_{0}(\eta)\right]+\hbar_{3} N_{3}[\breve{f}(\eta, q), \breve{g}(\eta, q), \breve{k}(\eta, q), \breve{h}(\eta, q)]\right]=0 \\
L_{2}\left[\breve{h}(\eta, q)-h_{0}(\eta)\right]-q\left[L_{2}\left[\breve{h}(\eta, q)-h_{0}(\eta)\right]+\hbar_{4} N_{4}[\breve{k}(\eta, q), \breve{g}(\eta, q), \breve{k}(\eta, q), \breve{h}(\eta, q)]\right]=0
\end{gathered}
$$

where,

$$
\begin{aligned}
& N_{1}[\breve{f}(\eta, q), \breve{g}(\eta, q)]=\frac{\partial^{3} \breve{f}(\eta, q)}{\partial \eta^{3}}-M \frac{\partial \breve{f}(\eta, q)}{\partial \eta}+2 \breve{f}(\eta, q) \frac{\partial^{2} \breve{f}(\eta, q)}{\partial \eta^{2}}+\alpha^{2}(\breve{g}(\eta, q))^{2}-\left(\frac{\partial \breve{f}(\eta, q)}{\partial \eta}\right)^{2}+1 \\
& +W e\left(\begin{array}{c}
2\left(\frac{\partial^{2} \breve{f}(\eta, q)}{\partial \eta^{2}}\right)^{2}-2 \breve{f}(\eta, q) \frac{\partial^{4} \breve{f}(\eta, q)}{\partial \eta^{4}} \\
+\lambda\left(-\alpha^{2}\left(\frac{\partial \breve{g}(\eta, q)}{\partial \eta}\right)^{2}+\left(\frac{\partial^{2} \breve{f}(\eta, q)}{\partial \eta^{2}}\right)^{2}-2 \frac{\partial \breve{f}(\eta, q)}{\partial \eta} \frac{\partial^{3} \breve{f}(\eta, q)}{\partial \eta^{3}}\right)
\end{array}\right)
\end{aligned}
$$




$$
\begin{aligned}
& N_{2}[\breve{f}(\eta, q), \breve{g}(\eta, q)]=\frac{\partial^{2} \breve{g}(\eta, q)}{\partial \eta^{2}}-M \breve{g}(\eta, q)-2 \breve{g}(\eta, q) \frac{\partial \breve{f}(\eta, q)}{\partial \eta}+2 \frac{\partial \breve{g}(\eta, q)}{\partial \eta} \breve{f}(\eta, q) \\
& +W e\left(2 \frac{\partial \breve{g}(\eta, q)}{\partial \eta} \frac{\partial^{2} \breve{f}(\eta, q)}{\partial \eta^{2}}-2 \breve{f}(\eta, q) \frac{\partial^{3} \breve{g}(\eta, q)}{\partial \eta^{3}}+\lambda\left(2 \frac{\partial \breve{g}(\eta, q)}{\partial \eta} \frac{\partial^{2} \breve{f}(\eta, q)}{\partial \eta^{2}}-2 \frac{\partial \breve{f}(\eta, q)}{\partial \eta} \frac{\partial^{2} \breve{g}(\eta, q)}{\partial \eta^{2}}\right)\right) \\
& N_{3}[\breve{f}(\eta, q), \breve{g}(\eta, q), \breve{k}(\eta, q), \breve{h}(\eta, q)]=\frac{\partial^{2} \breve{k}(\eta, q)}{\partial \eta^{2}}-M \breve{k}(\eta, q)+\alpha \breve{g}(\eta, q) \breve{h}(\eta, q)-\breve{k}(\eta, q) \frac{\partial \breve{f}(\eta, q)}{\partial \eta} \\
& +2 \breve{f}(\eta, q) \frac{\partial \breve{k}(\eta, q)}{\partial \eta}+W e\left(\begin{array}{c}
2 \frac{\partial \breve{k}(\eta, q)}{\partial \eta} \frac{\partial^{2} \breve{f}(\eta, q)}{\partial \eta^{2}}-\alpha \breve{h}(\eta, q) \frac{\partial^{2} \breve{g}(\eta, q)}{\partial \eta^{2}}+\alpha \breve{g}(\eta, q) \frac{\partial^{2} \breve{h}(\eta, q)}{\partial \eta^{2}} \\
-\frac{\partial \breve{f}(\eta, q)}{\partial \eta} \frac{\partial^{2} \breve{k}(\eta, q)}{\partial \eta^{2}}+\breve{k}(\eta, q) \frac{\partial^{3} \breve{f}(\eta, q)}{\partial \eta^{3}}-2 \breve{f}(\eta, q) \frac{\partial^{3} \breve{k(\eta, q)}}{\partial \eta^{3}} \\
+\lambda\left(-\alpha \frac{\partial \breve{g}(\eta, q)}{\partial \eta} \frac{\partial \breve{h}(\eta, q)}{\partial \eta}+\frac{\partial \breve{k}(\eta, q)}{\partial \eta} \frac{\partial^{2} \breve{f}(\eta, q)}{\partial \eta^{2}}-2 \frac{\partial \breve{f}(\eta, q)}{\partial \eta} \frac{\partial^{2} \breve{k}(\eta, q)}{\partial \eta^{2}}\right)
\end{array}\right) \\
& N_{4}[\breve{f}(\eta, q), \breve{g}(\eta, q), \breve{k}(\eta, q), \breve{h}(\eta, q)]=\frac{\partial^{2} \breve{h}(\eta, q)}{\partial \eta^{2}}-M \breve{h}(\eta, q)-\alpha \breve{g}(\eta, q) \breve{k}(\eta, q)-\breve{h}(\eta, q) \frac{\partial \breve{f}(\eta, q)}{\partial \eta} \\
& +2 \breve{f}(\eta, q) \frac{\partial \breve{h}(\eta, q)}{\partial \eta}+W e\left(\begin{array}{l}
2 \frac{\partial \breve{h}(\eta, q)}{\partial \eta} \frac{\partial^{2} \breve{f}(\eta, q)}{\partial \eta^{2}}+\alpha \breve{k}(\eta, q) \frac{\partial^{2} \breve{g}(\eta, q)}{\partial \eta^{2}}-\frac{\partial \breve{f}(\eta, q)}{\partial \eta} \frac{\partial^{2} \breve{h}(\eta, q)}{\partial \eta^{2}} \\
-\alpha \breve{g}(\eta, q) \frac{\partial^{2} \breve{k}(\eta, q)}{\partial \eta^{2}}+\breve{h}(\eta, q) \frac{\partial^{3} \breve{f}(\eta, q)}{\partial \eta^{3}}-2 \breve{f}(\eta, q) \frac{\partial^{3} \breve{h}(\eta, q)}{\partial \eta^{3}} \\
+\lambda\left(\begin{array}{l}
\alpha \frac{\partial \breve{g}(\eta, q)}{\partial \eta} \frac{\partial \breve{k}(\eta, q)}{\partial \eta} \\
+\frac{\partial \breve{h}(\eta, q)}{\partial \eta} \frac{\partial^{2} \widetilde{f}(\eta, q)}{\partial \eta^{2}}-2 \frac{\partial^{2} \breve{h}(\eta, q)}{\partial \eta^{2}} \frac{\partial \widetilde{f}(\eta, q)}{\partial \eta}
\end{array}\right)
\end{array}\right)
\end{aligned}
$$

in which, $q \in[0,1]$ is the embedding parameter and $\hbar$ is the non- zero auxiliary parameter.

The general HAM equations for $m t h$ order can be given by:

$$
\begin{aligned}
& L_{1}\left[f_{m}(\eta)-\chi_{m} f_{m-1}(\eta)\right]=\hbar_{1} R_{1, m}(\eta) \\
& L_{2}\left[g_{m}(\eta)-\chi_{m} g_{m-1}(\eta)\right]=\hbar_{2} R_{2, m}(\eta) \\
& L_{2}\left[k_{m}(\eta)-\chi_{m} k_{m-1}(\eta)\right]=\hbar_{3} R_{3, m}(\eta) \\
& L_{2}\left[h_{m}(\eta)-\chi_{m} h_{m-1}(\eta)\right]=\hbar_{4} R_{4, m}(\eta)
\end{aligned}
$$

with the following boundary conditions

$$
\begin{gathered}
\left(f_{m}(0), f_{m}^{\prime}(0), g_{m}(0), k_{m}(0), h_{m}(0)\right)=(0,0,0,0,0), \\
\left(f_{m}^{\prime}(\infty), g_{m}(\infty), k_{m}(\infty), h_{m}(\infty)\right)=(0,0,0,0) \\
\chi_{m}=\left\{\begin{array}{l}
0, m \leq 1 \\
1, m \geq 2
\end{array}\right.
\end{gathered}
$$




$$
\begin{aligned}
& R_{1, m}(\eta)=f^{\prime \prime \prime}{ }_{m-1}(\eta)-M f^{\prime}{ }_{m-1}(\eta) \\
& +W e \sum_{i=0}^{m-1}\left(2{f^{\prime \prime}}_{i}(\eta) f^{\prime \prime}{ }_{m-1-i}(\eta)-2 f_{i}(\eta){f^{\prime \prime \prime \prime}}_{m-1-i}(\eta)+\lambda\left(\begin{array}{l}
f^{\prime \prime}{ }_{i}(\eta){f^{\prime \prime}}_{m-1-i}(\eta) \\
-\alpha^{2}{g^{\prime}}_{i}(\eta){g^{\prime}}_{m-1-i}(\eta)-2{f^{\prime}}_{i}(\eta){f^{\prime \prime \prime}}_{m-1-i}(\eta)
\end{array}\right)\right) \\
& +2 f_{i}(\eta) f^{\prime \prime}{ }_{m-1-i}(\eta)+\alpha^{2} g_{i}(\eta) g_{m-1-i}(\eta)-f^{\prime}{ }_{i}(\eta) f^{\prime}{ }_{m-1-i}(\eta)+\left(1-\chi_{m}\right), \\
& R_{2, m}(\eta)=g^{\prime \prime}{ }_{m-1}(\eta)-M g_{m-1}(\eta)+W e \sum_{i=0}^{m-1}\left(\begin{array}{c}
2 g^{\prime}{ }_{i}(\eta) f^{\prime \prime}{ }_{m-1-i}(\eta)-2 f_{i}(\eta) g^{\prime \prime \prime}{ }_{m-1-i}(\eta) \\
+\lambda\left(2 g_{i}^{\prime}(\eta) f^{\prime \prime}{ }_{m-1-i}(\eta)-2{f^{\prime}}_{i}^{\prime}(\eta) g^{\prime \prime}{ }_{m-1-i}(\eta)\right)
\end{array}\right) \\
& -2 g_{i}(\eta) f_{m-1-i}^{\prime}(\eta)+2 g^{\prime}{ }_{i}(\eta) f_{m-1-i}(\eta)
\end{aligned}
$$$$
R_{3, m}(\eta)=k_{m-1}^{\prime \prime}(\eta)-M k_{m-1}(\eta)+W e \sum_{i=0}^{m-1}\left(\begin{array}{c}
2{k^{\prime}}_{i}(\eta) f^{\prime \prime}{ }_{m-1-i}(\eta)-\alpha h_{i}(\eta) g_{m-1-i}^{\prime \prime}(\eta)+\alpha g_{i}(\eta) h^{\prime \prime}{ }_{m-1-i}(\eta) \\
-f_{i}^{\prime}(\eta){k^{\prime \prime}}_{m-1-i}(\eta)+k_{i}(\eta) f^{\prime \prime \prime}{ }_{m-1-i}(\eta)-2 f_{i}(\eta){k^{\prime \prime \prime}}_{m-1-i}(\eta) \\
+\lambda\left(-\alpha g_{i}^{\prime}(\eta) h_{m-1-i}^{\prime}(\eta)+{k_{i}^{\prime}}_{i}(\eta){f^{\prime \prime}}_{m-1-i}(\eta)-2{f^{\prime}}_{i}(\eta){k^{\prime \prime}}_{m-1-i}(\eta)\right)
\end{array}\right)
$$$$
+\alpha g_{i}(\eta) h_{m-1-i}(\eta)-k_{i}(\eta) f_{m-1-i}^{\prime}(\eta)+2 f_{i}(\eta) k_{m-1-i}^{\prime}(\eta)
$$$$
R_{4, m}(\eta)=h^{\prime \prime}{ }_{m-1}(\eta)-\mathrm{M} h_{m-1}(\eta)+W e \sum_{i=0}^{m-1}\left(\begin{array}{c}
2{h^{\prime}}_{i}(\eta) f^{\prime \prime}{ }_{m-1-i}(\eta)+\alpha k_{i}(\eta) g^{\prime \prime}{ }_{m-1-i}(\eta)-f_{i}^{\prime}(\eta) h^{\prime \prime}{ }_{m-1-i}(\eta) \\
-\alpha g_{i}(\eta){k^{\prime \prime}}_{m-1-i}(\eta)+h_{i}(\eta) f^{\prime \prime \prime}{ }_{m-1-i}(\eta)-2 f_{i}(\eta) h^{\prime \prime \prime}{ }_{m-1-i}(\eta) \\
+\lambda\left(\alpha g_{i}^{\prime}(\eta) k_{m-1-i}^{\prime}(\eta)+h_{i}^{\prime}(\eta) f^{\prime \prime}{ }_{m-1-i}(\eta)-2 h^{\prime \prime}{ }_{i}(\eta) f^{\prime}{ }_{m-1-i}(\eta)\right)
\end{array}\right)
$$$$
-\alpha g_{i}(\eta) k_{m-1-i}(\eta)-h_{i}(\eta) f_{m-1-i}^{\prime}(\eta)+2 f_{i}(\eta) h_{m-1-i}^{\prime}(\eta)
$$

According to the above defined method, the linear Eqs. (38)-(41) in the order $m=1,2,3, \ldots$, can be solved by means of a computational software Mathematica.

\section{Graphical results and discussion}

In previous sections the governing equations with the boundary conditions have been solved in order to give the details of flow fields. In the present section, the effects of key parameters, which emerge in the governing equations have been highlighted by comparison and discussion on velocity profiles of the fluid.

\subsection{Convergence analysis}

It is very significant to establish the convergence and accuracy of an analytical solution. The convergence and rate of approximation of the HAM solutions rely upon the value of auxiliary parameter $\hbar$. The homotopy approach with auxiliary parameter $\hbar$ gives the freedom to choose the convergence regions for the attained solutions. Plotting of graphs for $f^{\prime \prime}(0), g^{\prime}(0), k^{\prime}(0)$ and $h^{\prime}(0)$ against the auxiliary parameters $\hbar_{1}, \hbar_{2}, \hbar_{3}$ and $\hbar_{4}$ provide the area in which the obtained solutions can converge. The possible values for these auxiliary parameters are $-0.3<\hbar_{1}<0.1,-0.25<\hbar_{2}<0.2$, $-0.3<\hbar_{3}<0.1$, and $-0.3<\hbar_{4}<0.15$ that has been shown in Fig. 26. Figure demonstrates the permissible regions for $\hbar_{1}, \hbar_{2}, \hbar_{3}, \hbar_{4}$ coincide with the line segments which are along the horizontal axis. To obtain better understanding, 
Table 4 illustrates the values of well-founded regions. By selecting the best possible value of auxiliary parameter, the averaged residual errors for each approximated solution are described as [22].

$$
\begin{aligned}
& E_{1, m}=\frac{1}{\mathrm{~N}} \sum_{j=1}^{\mathrm{N}}\left\{N_{f}\left[\sum_{i=0}^{m} f_{i}(j \delta)\right]\right\}^{2} \\
& E_{2, m}=\frac{1}{\mathrm{~N}} \sum_{j=1}^{\mathrm{N}}\left\{N_{g}\left[\sum_{i=0}^{\mathrm{m}} g_{i}(j \delta)\right]\right\}^{2} \\
& E_{3, m}=\frac{1}{\mathrm{~N}} \sum_{j=1}^{\mathrm{N}}\left\{N_{k}\left[\sum_{i=0}^{m} k_{i}(j \delta)\right]\right\}^{2} \\
& E_{4, m}=\frac{1}{\mathrm{~N}} \sum_{j=1}^{\mathrm{N}}\left\{N_{h}\left[\sum_{i=0}^{m} h_{i}(j \delta)\right]\right\}^{2}
\end{aligned}
$$

where $\mathrm{N}$ represents the equally distributed discrete points, therefore, $\delta=\frac{10}{\mathrm{~N}}$ and $\mathrm{N}=20$. According to the given order of approximation $m$, the optimal values of $\hbar_{1}, \hbar_{2}, \hbar_{3}$, and $\hbar_{4}$ can be determined by minimizing the residual errors relating to the nonlinear algebraic equations as

$$
\frac{d_{1, m}}{d \hbar_{1}}=0, \frac{d_{2, m}}{d \hbar_{2}}=0, \frac{d_{3, m}}{d \hbar_{3}}=0, \frac{d_{4, m}}{d \hbar_{4}}=0 .
$$

To acquire the accuracy corresponding to the solutions of nonlinear equations, the residual errors can be interpreted for the system as

$$
\begin{gathered}
\operatorname{Res}(f)=f^{\prime \prime \prime}(\eta)-M f^{\prime}(\eta)+2 f(\eta) f^{\prime \prime}(\eta)+\alpha^{2} g^{2}(\eta)-f^{\prime 2}(\eta)+1 \\
+W e\left(2 f^{\prime \prime 2}(\eta)-2 f(\eta) f^{\prime \prime \prime \prime}(\eta)+\lambda\left(-\alpha^{2} g^{\prime 2}(\eta)+f^{\prime \prime 2}(\eta)-2 f^{\prime}(\eta) f^{\prime \prime \prime}(\eta)\right)\right), \\
\operatorname{Res}(g)=g^{\prime \prime}(\eta)-M g(\eta)-2 g(\eta) f^{\prime}(\eta)+2 g^{\prime}(\eta) f(\eta)+W e\left(\begin{array}{c}
2 g^{\prime}(\eta) f^{\prime \prime}(\eta)-2 f(\eta) g^{\prime \prime \prime}(\eta) \\
+\lambda\left(2 g^{\prime}(\eta) f^{\prime \prime}(\eta)-2 f^{\prime}(\eta) g^{\prime \prime}(\eta)\right)
\end{array}\right), \\
\operatorname{Res}(k)=k^{\prime \prime}(\eta)-M k(\eta)+\alpha g(\eta) h(\eta)-k(\eta) f^{\prime}(\eta)+2 f(\eta) k^{\prime}(\eta) \\
+W e\left(\begin{array}{l}
2 k^{\prime}(\eta) f^{\prime \prime}(\eta)-\alpha h(\eta) g^{\prime \prime}(\eta)+\alpha g(\eta) h^{\prime \prime}(\eta)-f^{\prime}(\eta) k^{\prime \prime}(\eta) \\
+k f^{\prime \prime \prime}(\eta)-2 f(\eta) k^{\prime \prime \prime}(\eta)+\lambda\left(k^{\prime}(\eta) f^{\prime \prime}(\eta)-\alpha g^{\prime}(\eta) h^{\prime}(\eta)-2 f^{\prime}(\eta) k^{\prime \prime}(\eta)\right)
\end{array}\right), \\
\operatorname{Res}(h)=h^{\prime \prime}(\eta)-M h(\eta)-\alpha g(\eta) k(\eta)-h(\eta) f^{\prime}(\eta)+2 f(\eta) h^{\prime}(\eta) \\
+W e\left(\begin{array}{l}
2 h^{\prime}(\eta) f^{\prime \prime}(\eta)+\alpha k(\eta) g^{\prime \prime}(\eta)-f^{\prime}(\eta) h^{\prime \prime}(\eta)-\alpha g(\eta) k^{\prime \prime}(\eta) \\
+h(\eta) f^{\prime \prime \prime}(\eta)-2 f(\eta) h^{\prime \prime \prime}(\eta)+\lambda\left(h^{\prime}(\eta) f^{\prime \prime}(\eta)+\alpha g^{\prime}(\eta) k^{\prime}(\eta)-2 h^{\prime \prime}(\eta) f^{\prime}(\eta)\right)
\end{array}\right)
\end{gathered}
$$

The main inadequacy of the errors given in (48) - (51) is that the cost to compute them may not be coincide with our desire, especially in unbounded problems. In view of these shortcomings, by using ratio $\beta=\frac{\left\|\mathbf{u}_{i+1}\right\|}{\left\|\mathbf{u}_{i}\right\|}$, providing a simple way of calculating the values of $\hbar_{1}, \hbar_{2}, \hbar_{3}$, and $\hbar_{4}$ proposed in [23-25]. The Figs. $27 \mathrm{a}-27 \mathrm{~d}$ illustrate the $\beta$-curves against the number of successive terms $i$, which show that the finite values of $\beta$ are obtained by going towards $i \rightarrow \infty$, which are always less than unity. This test will play an important role for checking the convergence of the solutions obtained by HAM towards the exact solution. 


\subsection{Comparison analysis}

The numerical comparison of various velocity components has been noticed in Table 1 for increasing values of rotational parameter $\alpha$ keeping the values of $W e, \lambda, M, f_{w}$, and $\hbar$ unchanged i.e. $\left(W e=\lambda=M=f_{w}=0, \hbar=-0.25\right)$ which converts the system to the Newtonian fluid problem of Wang [9]. Also, the present solutions have been verified by comparing the results obtained by HAM (as a special case i.e. $\left(W e=\lambda=M=f_{w}=0\right)$ ) with the solutions of Newtonian fluid flow for an off-centered rotating disk which had been reported by Wang [9] as see in Figs. $22-25$. The identical approach has been observed between Wang [9] and present analytic solutions of HAM.

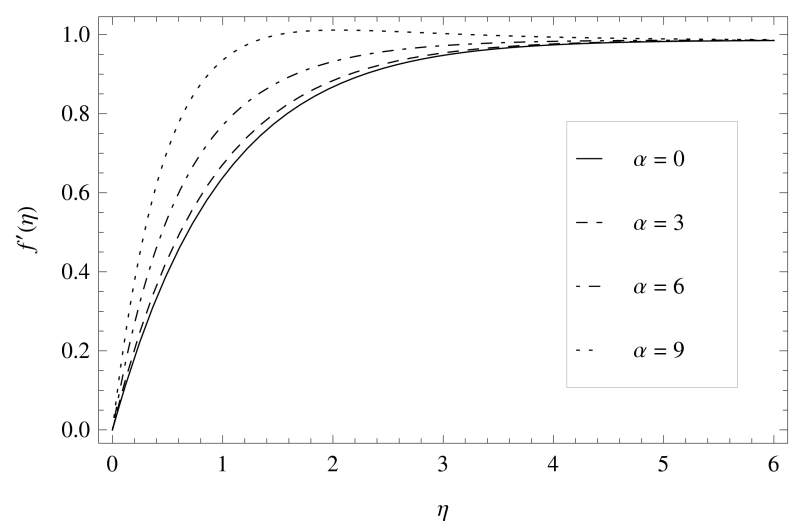

Fig. 2: The influence of a parameter $\alpha$ on $f^{\prime}(\eta)$ for $\lambda=0.01, W e=$ $0.2, M=0.3, f_{w}=0.01$ and $\hbar=-0.01$.

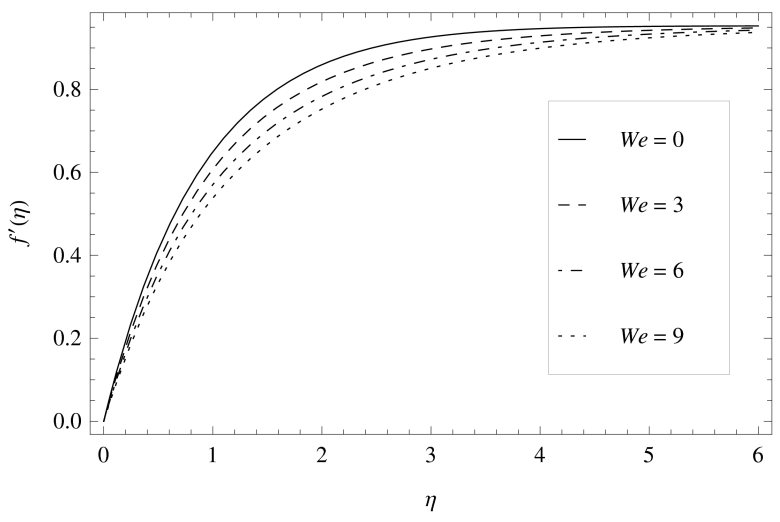

Fig. 3: The influence of a parameter We on $f^{\prime}(\eta)$ for $\alpha=2, \lambda=$ $0.5, M=1, f_{w}=0.7$ and $\hbar=-0.01$

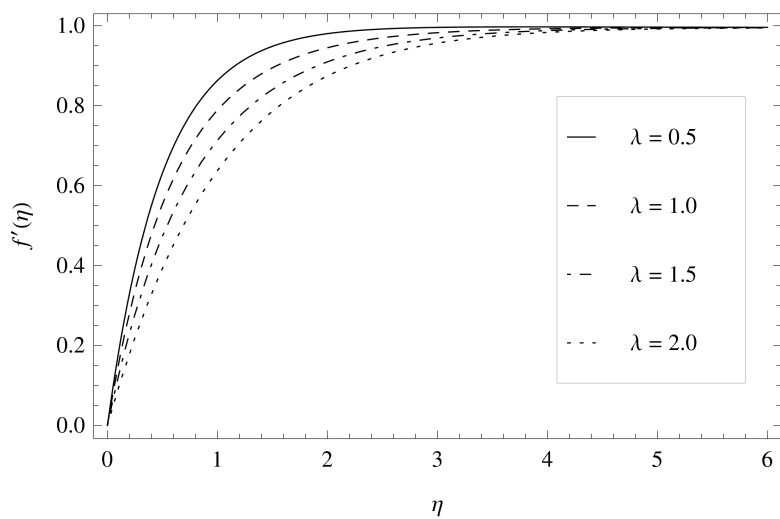

Fig. 4: The influence of a parameter $\lambda$ on $f^{\prime}(\eta)$ for $\alpha=9$, We $=$ $0.5, M=0.1, f_{w}=0.4$ and $\hbar=-0.01$

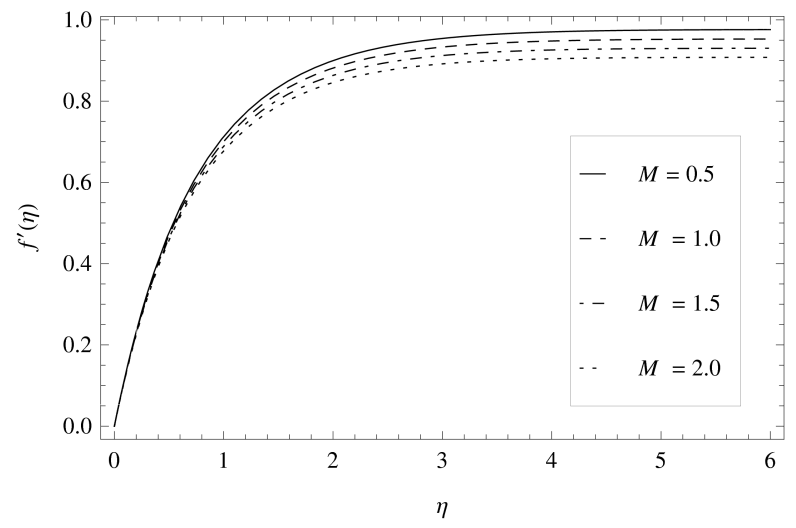

Fig. 5: The influence of a parameter $M$ on $f^{\prime}(\eta)$ for $\alpha=4.5, \lambda=$ $0.1, W e=0.3, f_{w}=0.8, \hbar=-0.01$

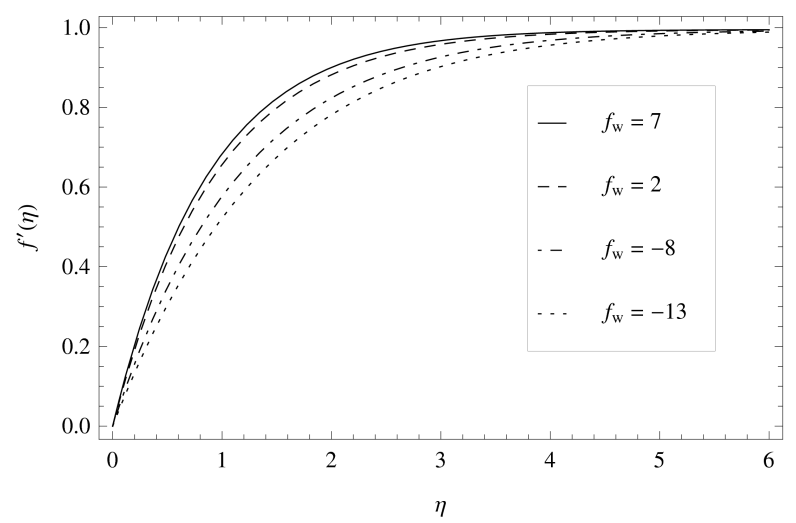

Fig. 6: The influence of a parameter $f_{w}$ on $f^{\prime}(\eta)$ for $W e=0.6, \alpha=$ $0.1, M=0.1, \lambda=0.1$ and $\hbar=-0.01$

\subsection{Flow Characteristics}

In the interest to obtain a physical insight into the problem, velocity distribution has been discussed by allowing admissible values of various main controlling param- 
Table 1: Illustrating the variation of $f^{\prime \prime}(0) g^{\prime}(0) k^{\prime}(0)$, and $h^{\prime}(0)$ for various values of $\alpha$ at the approximation level $m=9(W e=0, \lambda=0$, $\left.M=0, f_{w}=0, \hbar=-0.25\right)$

\begin{tabular}{cccccc}
\hline$\alpha$ & 0 & 0.5 & 1 & 2 & 3 \\
\hline$f^{\prime \prime}(0)$ Wang [9] HAM & 1.31196 & 1.37875 & 1.57393 & 2.29491 & 3.25656 \\
& 1.31253 & 1.32757 & 1.50440 & 2.17375 & 3.16203 \\
$g^{\prime}$ (0) Wang [9] HAM & -1.0747 & -1.08393 & -1.11002 & -1.19918 & -1.41529 \\
& -1.06507 & -1.07095 & -1.16841 & -1.17523 & -1.23597 \\
$k^{\prime}$ (0) Wang [9] HAM & 0 & 0.137977 & 0.270021 & 0.501004 & 0.596779 \\
& 0.02242 & 0.13922 & 0.31488 & 0.50645 & 0.78049 \\
$h^{\prime}(0)$ Wang [9] HAM & -0.938803 & -0.949555 & -0.979767 & -1.08333 & -1.33644 \\
& -0.928891 & -0.95039 & -0.983592 & -1.07848 & -1.19686 \\
\hline
\end{tabular}

Table 2: Illustrating the variation of $f^{\prime \prime}(0)$ for various $W e$ and $\lambda$ at the approximation level $m=9,\left(\hbar=-0.29, \alpha=0, M=0, f_{W}=0\right)$

\begin{tabular}{crllll}
\hline$W e \lambda$ & \multicolumn{1}{c}{0} & \multicolumn{1}{c}{0.1} & \multicolumn{1}{c}{0.2} & \multicolumn{1}{c}{0.3} & \multicolumn{1}{c}{0.4} \\
\hline 0 & 1.30776 & 1.32607 & 1.38353 & 1.44003 & 1.49548 \\
0.1 & 1.30776 & 1.33449 & 1.40118 & 1.46766 & 1.53376 \\
0.2 & 1.30776 & 1.3430 & 1.41925 & 1.49626 & 1.57382 \\
0.3 & 1.30776 & 1.35163 & 1.43775 & 1.52588 & 1.61574 \\
0.4 & 1.30776 & 1.36035 & 1.4567 & 1.55655 & 1.6596 \\
\hline
\end{tabular}

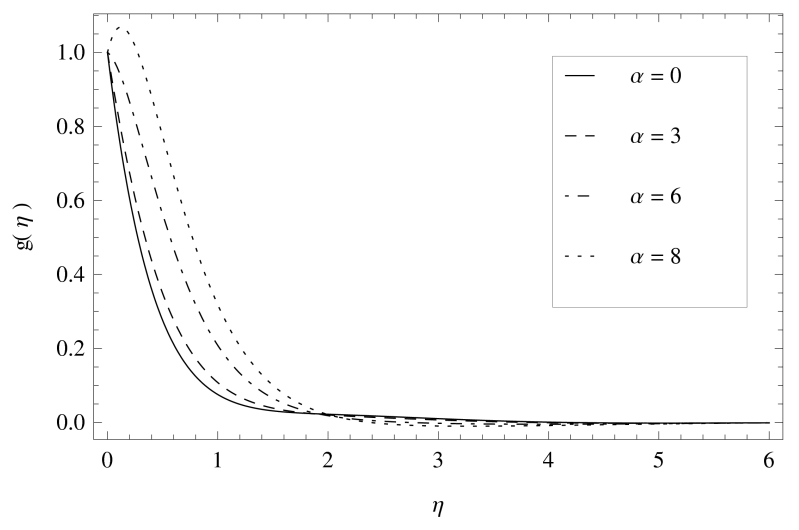

Fig. 7: The influence of a parameter $\alpha$ on $g(\eta)$ for $W e=0.9, \lambda=$ $2, M=3, f_{w}=0.2$ and $\hbar=-0.1$

eters including rotational parameter $\alpha$, Weissenberg number $W e$, ratio parameter $\lambda$, magnetic parameter $M$ and nondimensional velocity parameter $f_{w}$. The representative results for the velocity profiles at the $9^{\text {th }}$ order of approximation have been illustrated through Figs. 2 - 21 .

Figs. $2-6$ display the variations of the velocity component $f^{\prime}(\eta)$ in the radial direction pertaining to the various values of the parameters $\alpha, W e, \lambda, M$ and $f_{w}$. It can be examined from Fig. 2 that an increment in the rotational parameter $\alpha$ increases the velocity component $f^{\prime}(\eta)$ and it supports the flow of the fluid in the radial direction. It also depicts that the velocity function $f^{\prime}(\eta)$ rises monotonically to the finite value of velocity due to the pressure gradient at infinity. Fig. 3 unveils that variation in the values of $W e$

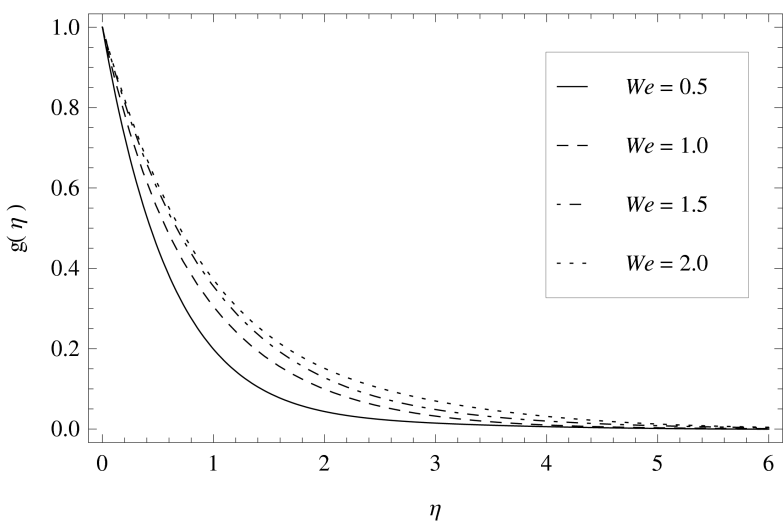

Fig. 8: The influence of a parameter We on $g(\eta)$ for $\lambda=0.5, \alpha=$ 2, $M=1, f_{w}=1.5$ and $\hbar=-0.1$

decreases $f^{\prime}(\eta)$, which illustrates that increment in elasticviscosity coefficient opposes the radial flow. It can be perceived from Fig. 4 that the velocity component $f^{\prime}(\eta)$ shows the decreasing effect when rising in the values of ratio of cross-viscosity and elastic-viscosity coefficients $\lambda$. The increment in the ratio of cross-viscosity and elastic-viscosity coefficients resists the flow in the radial direction due to an occurrence of rotation in the disk. From inspection of Fig. 5, it is observed that the increasing values of magnetic field parameter $M$ yield the conflicting behavior of velocity component $f^{\prime}(\eta)$ in radial direction and decreases the velocity. The effect of suction/injection parameter $f_{w}$ on radial velocity $f^{\prime}(\eta)$ can be anticipated from Fig. 6 . The values of $f_{w}<0$ correspond to injection of the fluid and it 
Table 3: Illustrating the variation of $f^{\prime \prime}(0)$ for various $M$ and $f_{w}$ at the approximation level $m=9(\hbar=-0.29, \alpha=0, W e=0, \lambda=0)$

\begin{tabular}{cccccc}
\hline$M \backslash f_{w}$ & 0 & 0.1 & 0.2 & 0.3 & 0.4 \\
\hline 0 & 1.30776 & 1.21822 & 1.17192 & 1.12863 & 1.08813 \\
0.1 & 1.36472 & 1.31089 & 1.26054 & 1.21342 & 1.16929 \\
0.2 & 1.46552 & 1.40725 & 1.3527 & 1.30162 & 1.25375 \\
0.3 & 1.57009 & 1.50724 & 1.44836 & 1.39317 & 1.34143 \\
0.4 & 1.67836 & 1.61078 & 1.54743 & 1.48802 & 1.43228 \\
\hline
\end{tabular}

Table 4: The values of optimal $\hbar_{1}, \hbar_{2}, \hbar_{3}, \hbar_{4}$ and maximum residual errors (MaxRes) at various values of $\alpha\left(W e=0.04, \lambda=0.04, f_{w}=\right.$ $0.01, M=0.6)$

\begin{tabular}{lllll}
\hline$\alpha$ & $\left(\hbar_{1}\right.$, MaxRes $)$ & $\left(\hbar_{2}\right.$, MaxRes $)$ & $\left(\hbar_{3}\right.$, MaxRes $)$ & $\left(\hbar_{4}\right.$, MaxRes $)$ \\
\hline \multirow{2}{*}{0.3} & $(-0.2708$, & $(-0.2321$, & $(-0.301$, & $(-0.250$, \\
& $\left.1.928 \times 10^{-5}\right)$ & $\left.5.081 \times 10^{-6}\right)$ & $\left.2.360 \times 10^{-8}\right)$ & $\left.1.275 \times 10^{-9}\right)$ \\
0.5 & $(-0.2492$, & $(-0.2150$, & $(-0.292$, & $(-0.231$, \\
& $\left.2.574 \times 10^{-7}\right)$ & $\left.6.149 \times 10^{-8}\right)$ & $\left.7.164 \times 10^{-8}\right)$ & $\left.3.147 \times 10^{-10}\right)$ \\
0.7 & $(-0.2183$, & $(-0.2131$, & $(-0.241$, & $(-0.215$, \\
& $\left.2.981 \times 10^{-8}\right)$ & $\left.1.321 \times 10^{-9}\right)$ & $\left.4.845 \times 10^{-9}\right)$ & $\left.5.319 \times 10^{-11}\right)$ \\
0.9 & $(-0.2161$, & $(-0.2014$, & $(-0.219$, & $(-0.203$, \\
& $\left.4.275 \times 10^{-8}\right)$ & $\left.5.341 \times 10^{-11}\right)$ & $\left.3.214 \times 10^{-11}\right)$ & $\left.6.217 \times 10^{-12}\right)$ \\
\hline
\end{tabular}

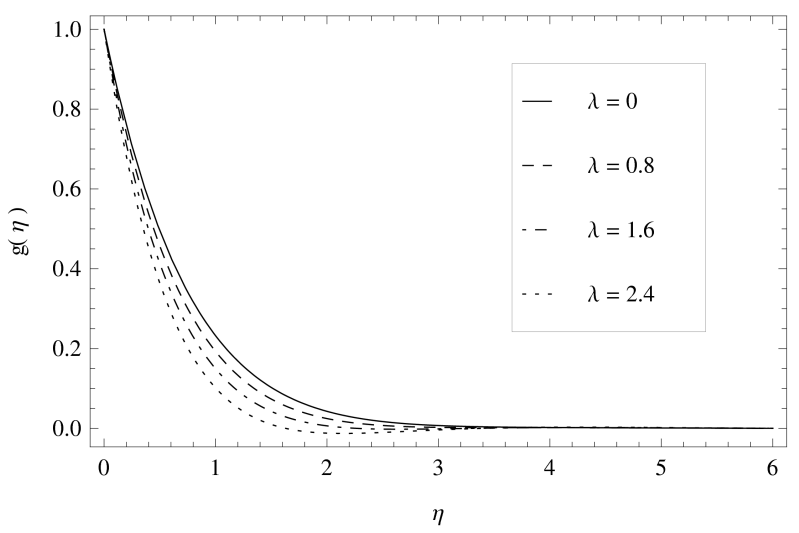

Fig. 9: The influence of a parameter $\lambda$ on $g(\eta)$ for $\alpha=0.07$, We $=$ $0.4, M=1.5, f_{w}=0.1$ and $\hbar=-0.1$

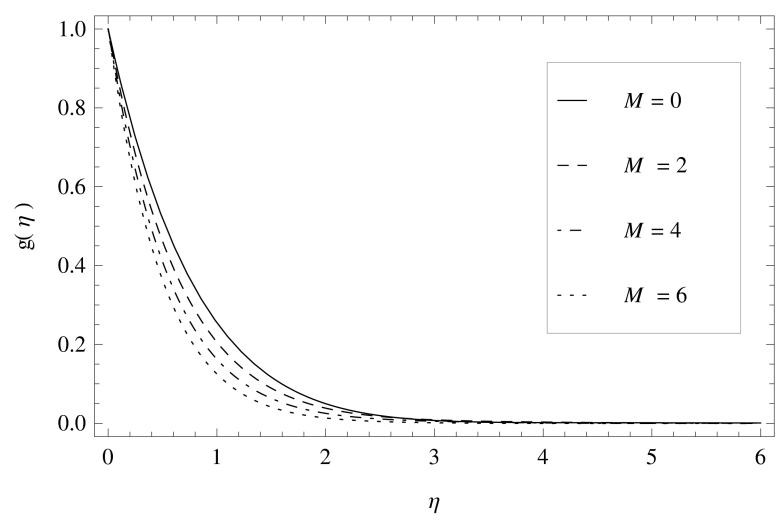

Fig. 10: The influence of a parameter $M$ on $g(\eta)$ for $W e=0.5, \alpha=$ $2, \lambda=0.2, f_{w}=0.2$ and $\hbar=-0.1$

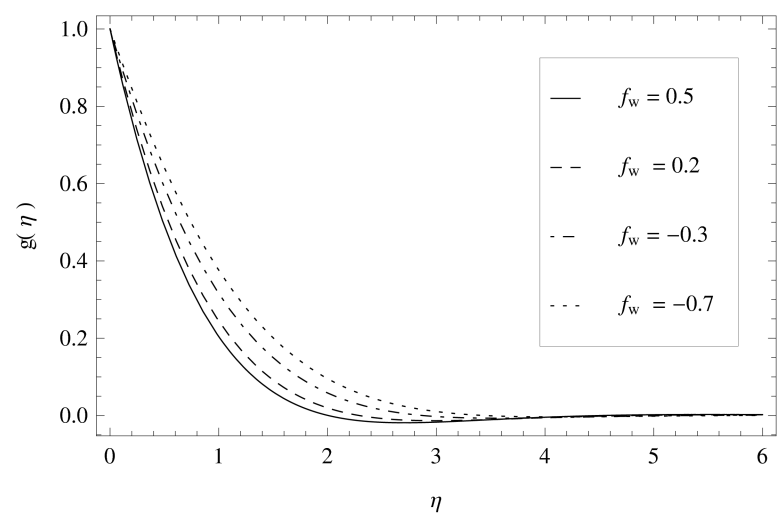

Fig. 11: The influence of a parameter $f_{w}$ on $g(\eta)$ for $\lambda=0.4$, We $=$ $0.1, \alpha=0.3, M=0.2$ and $\hbar=-0.1$

predicts that $f^{\prime}(\eta)$ increases with the increasing values of $f_{w}$, while the values of $f_{w}>0$ represent suction and it is also showing the same increasing behavior of $f^{\prime}(\eta)$ with increasing the values of $f_{w}$. Figs. $7-11$ present the effects of non-dimensional parameters on azimuthal velocity component $g(\eta)$. It is examined from Fig. 7 that the fluid particles stimulate towards the azimuthal direction as the rotation of the disk surface is increased which developes the increasing behavior of velocity component $g(\eta)$ in the azimuthal direction. Fig. 8 displays that the variation in the parameter We supports the flow in the azimuthal direction and increases the fluid motion by increasing the values of $W e$. It can be inferred from Fig. 9 that the velocity function $g(\eta)$ decreases as increasing the values of $\lambda$ which shows that the rising in the values of cross viscosity pa- 


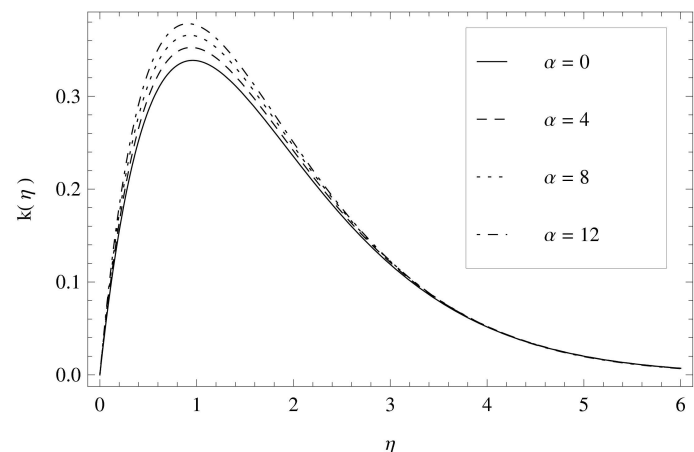

Fig. 12: The influence of a parameter $\alpha$ on $k(\eta)$ for $W e=0.2, \lambda=$ $0.1, M=0.4, f_{w}=0.7, \hbar=-0.01$

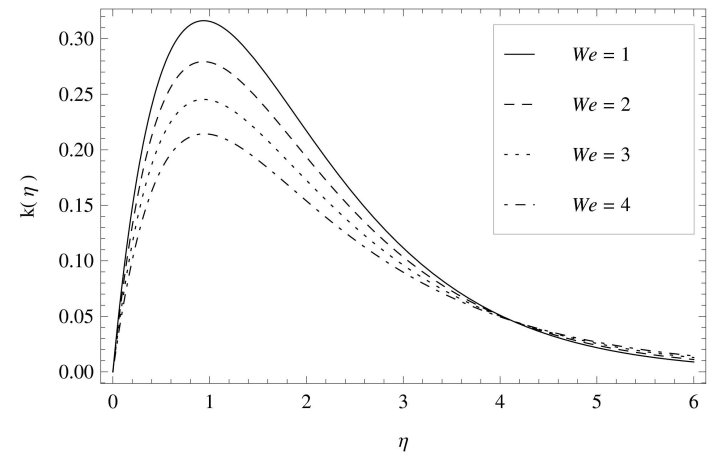

Fig. 13: The influence of a parameter We on $k(\eta)$ for $\lambda=0.1, \alpha=$ $3, M=0.4, f_{w}=0.9, \hbar=-0.01$

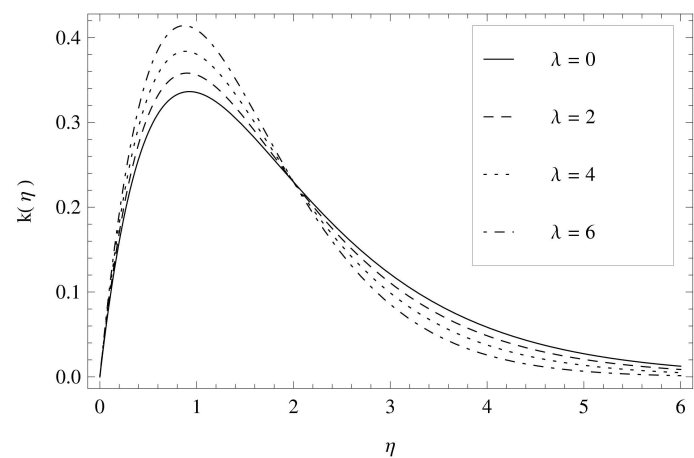

Fig. 14: The influence of a parameter $\lambda$ on $k(\eta)$ for $\alpha=1, W e=$ $2, M=0.01, f_{W}=0.01$ and $\hbar=-0.01$

rameter decelerate the fluid flow in the azimuthal direction. It is cleared from Fig.10 that as the strength of the magnetic field $M$ increases, the azimuthal velocity function $g(\eta)$ moves downward. This decreasing behavior ex-

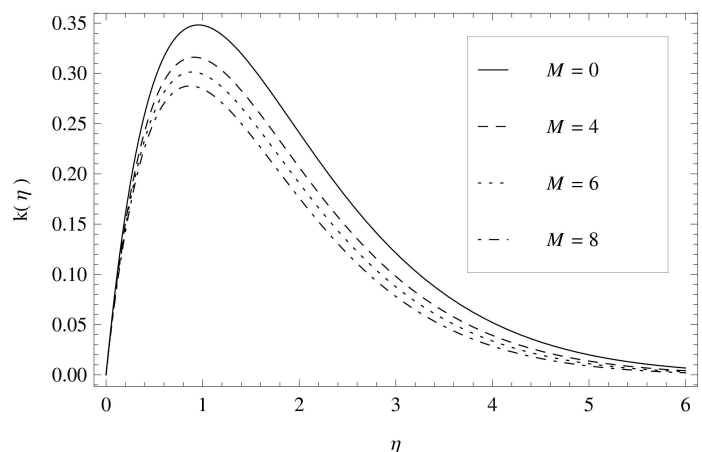

Fig. 15: The influence of a parameter $M$ on $k(\eta)$ for $\lambda=0.3, W e=$ $0.1, \alpha=1, f_{w}=0.9$ and $\hbar=-0.01$

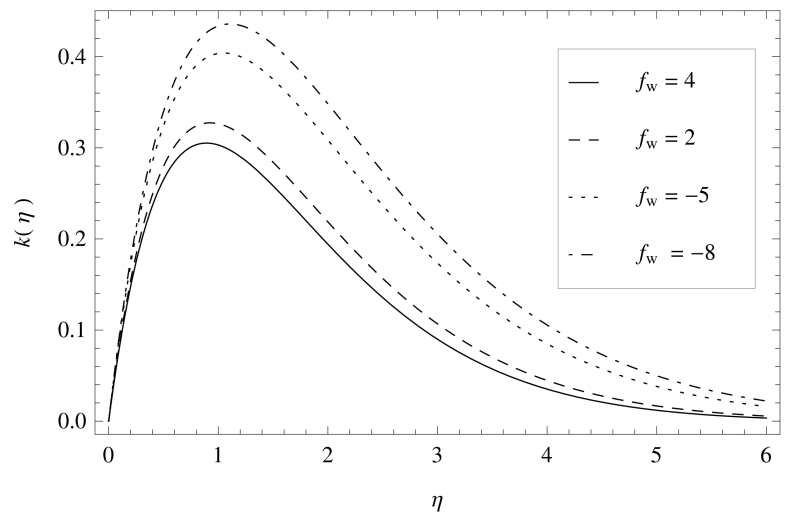

Fig. 16: The influence of a parameter $f_{w}$ on $k(\eta)$ for $\lambda=0.5, W e=$ $0.3, \alpha=2, M=0.5$ and $\hbar=-0.01$

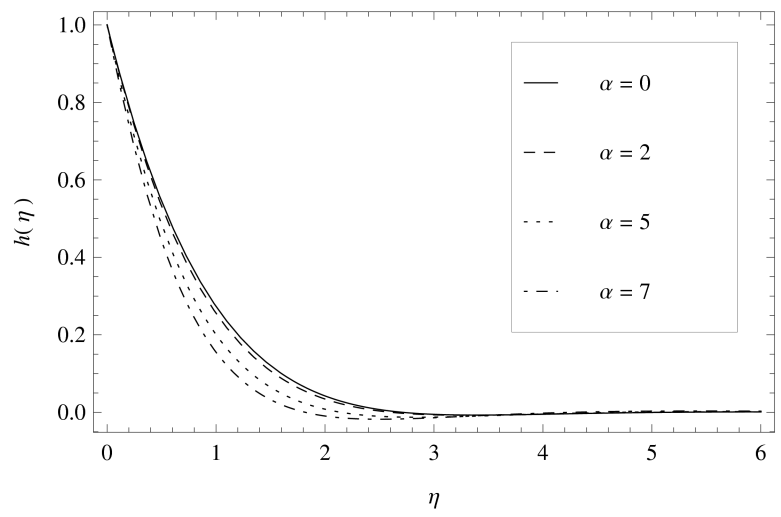

Fig. 17: The influence of a parameter $\alpha$ on $h(\eta)$ for $\lambda=0.4, W e=$ $0.2, M=0.3, f_{w}=0.09$ and $\hbar=-0.1$

hibits that the magnetic field parameter compels the velocity component $g(\eta)$ to provide us a conflict behavior in the azimuthal direction. It is tempting to notice that for an imposed magnetic field, the velocity component $g(\eta)$ decreases as suction is applied (see Fig. 11), while similar 


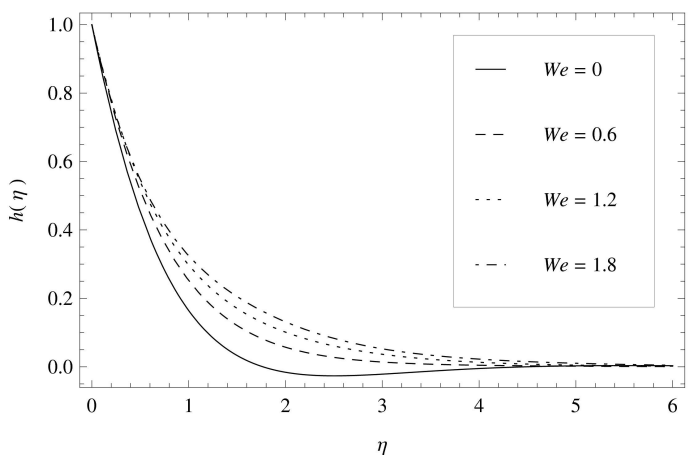

Fig. 18: The influence of a parameter We on $h(\eta)$ for $\lambda=0.1, \alpha=$ $3, M=0.6, f_{w}=0.5$ and $\hbar=-0.1$

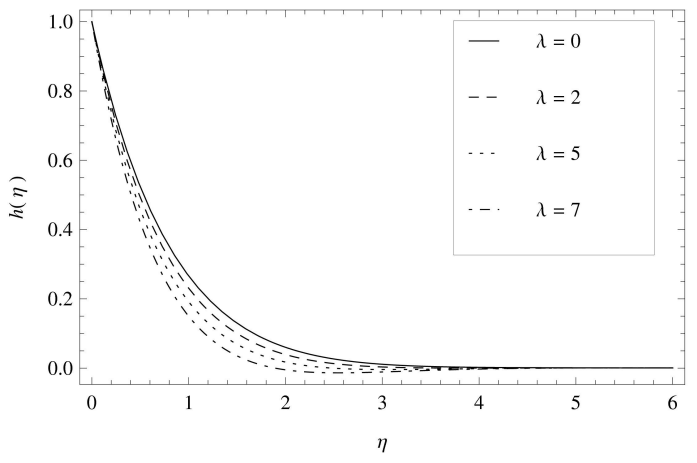

Fig. 19: The influence of a parameter $\lambda$ on $h(\eta)$ for $\alpha=0.1$, We $=$ $0.6, M=0.03, f_{w}=0.01$ and $\hbar=-0.1$

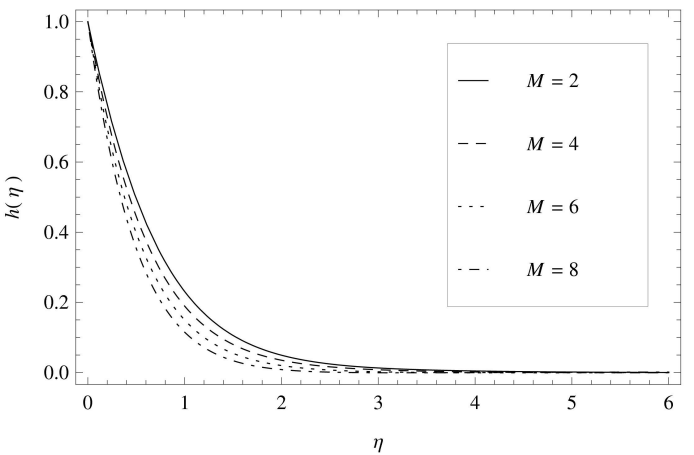

Fig. 20: The influence of a parameter $M$ on $h(\eta)$ for $\lambda=0.2, W e=$ $0.6, \alpha=6, f_{w}=0.1$ and $\hbar=-0.1$

effect is examined by increasing the injection velocity. It reveals that the intensity of fluid motion declines as raising the values of suction or injection velocities and the velocity function $g(\eta)$ becomes relatively constant near the

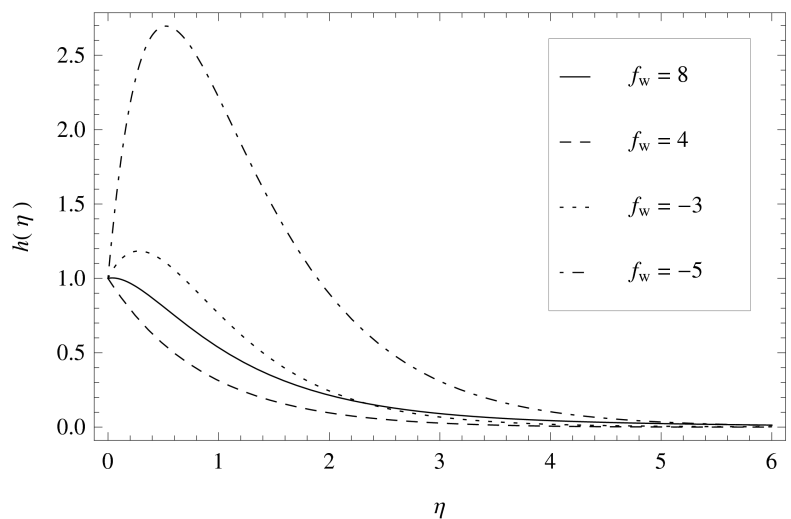

Fig. 21: The influence of a parameter $f_{w}$ on $h(\eta)$ for $\lambda=0.4, W e=$ $0.8, \alpha=2, M=0.7$ and $\hbar=-0.1$

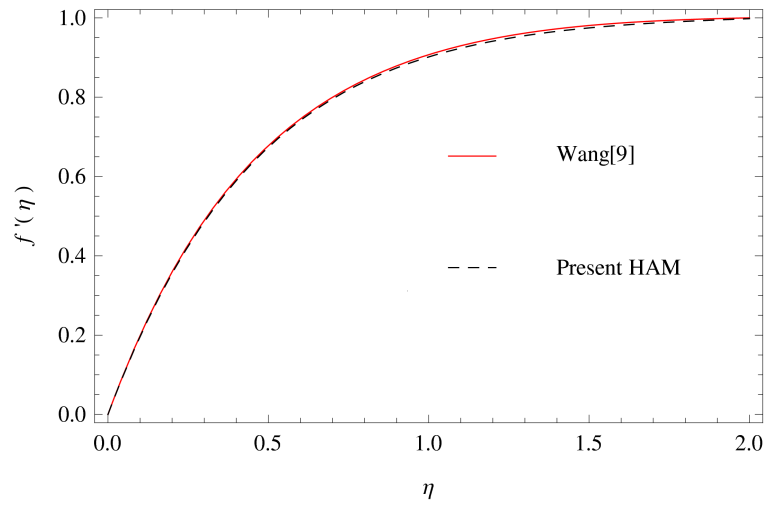

Fig. 22: Comparison of Wang [9] and HAM solution for velocity function $f^{\prime}(\eta)$

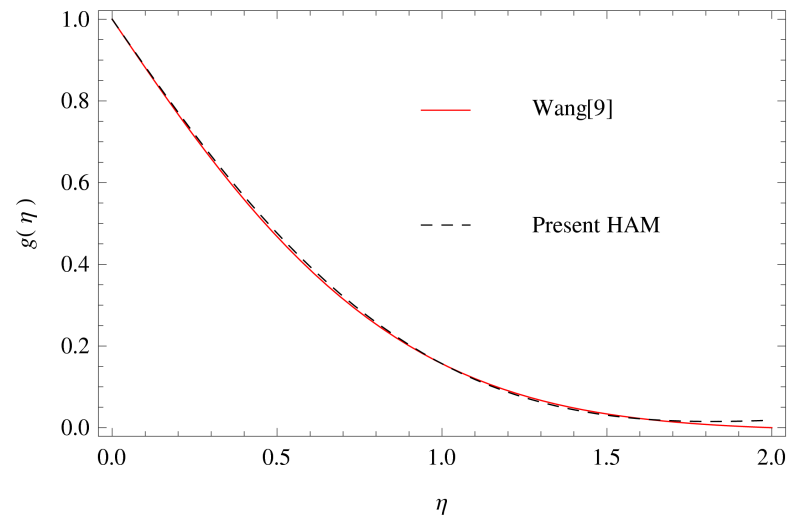

Fig. 23: Comparison of Wang [9] and HAM solution for velocity function $g(\eta)$

disk surface. The effects of parameters on an induced velocity component $k(\eta)$ can be perceived from Figs. $12-16$. Fig. 12 depicts that the increasing values of $\alpha$ rises $k(\eta)$ near the disk surface then decline exponentially and tends to zero as the values are approaching to infinity. The ve- 


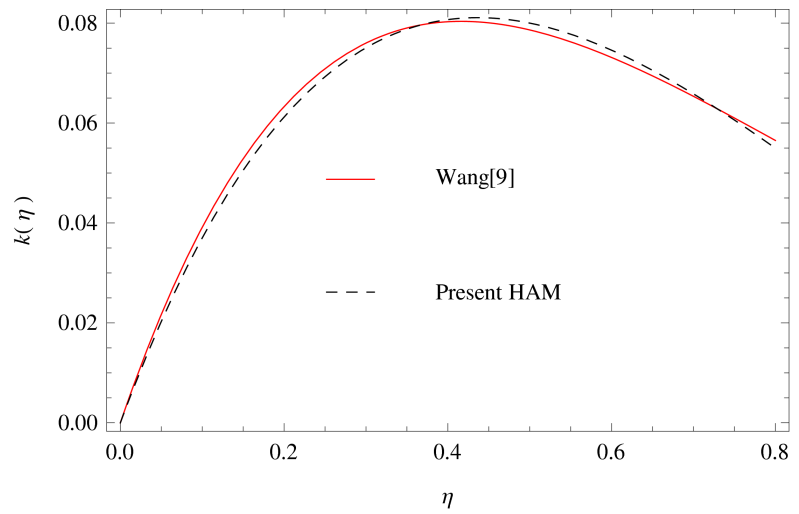

Fig. 24: Comparison of Wang [9] and HAM solution for velocity function $k(\eta)$

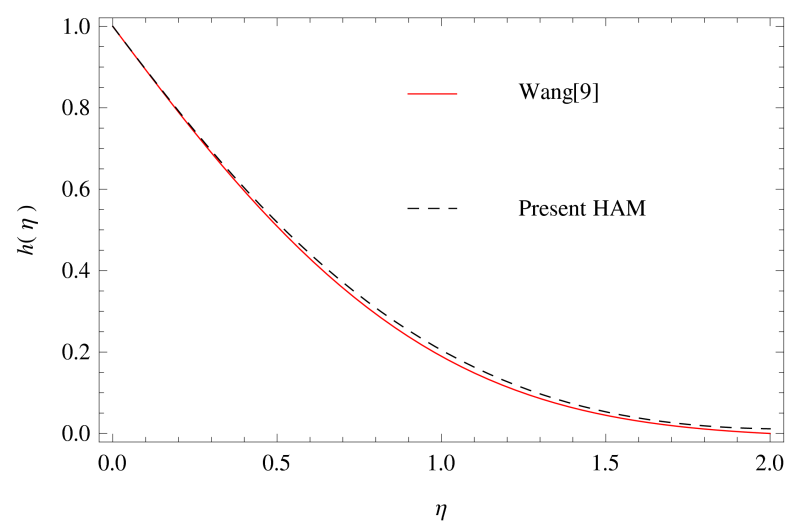

Fig. 25: Comparison of Wang [9] and HAM solution for velocity function $h(\eta)$

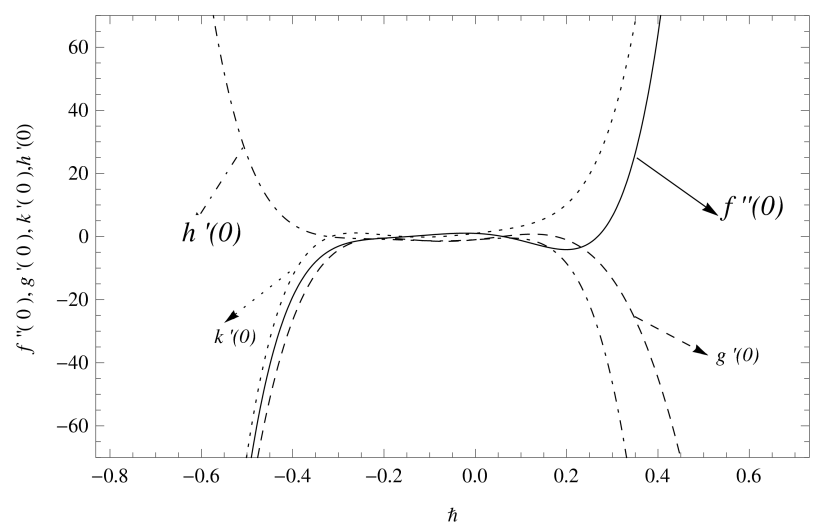

Fig. 26: The $\hbar$ curves vs. initial values of $f^{\prime \prime}(0), g^{\prime}(0), k^{\prime}(0)$ and $h^{\prime}(0)$

locity component $k(\eta)$ is decreased with an increment in Weissenberg number We as shown in Fig.13. It shows that the Weissenberg number which relies on elastic-viscous parameter is inversely related with the induced velocity. It can be interpreted form Fig.14 that the induced velocity $k(\eta)$ has a conflicting behavior with the ratio parameter $\lambda$. It rises with increasing the values of $\lambda$ near the disk and then starts to decline exponentially with large values of $\lambda$ which provides that the ratio parameter accelerates the velocity component initially but decreases as it tends to infinity. It can be seen from Fig. 15 that the strength of magnetic field $M$ has a negative impact on the velocity component $k(\eta)$ and it decreases the fluid flow as increasing the values of $M$. Fig. 16 shows that the increasing values of both suction/injection velocities $f_{w}$ tend to reduce the induced velocity $k(\eta)$ which leads to an idea that the velocity of the fluid is diminished by increasing the suction or injection parameter. The effects of pertinent parameters on induced velocity function $h(\eta)$ can be inferred from Figs. $17-21$. Fig. 17 illustrates that the increasing values of rotational parameter $\alpha$ provide impulsive pattern of flow. It decelerates the induced velocity $h(\eta)$ in the beginning but after some points, it enhances the intensity of the flow. It is exposed from Fig. 18 that as the values of We rises, it supports the motion of fluid and increases the velocity component $h(\eta)$. This increasing effect indicates that when we rise the elastic-viscous parameter on which the Weissenberg number relies on, it gives the stimulation in the induced velocity component. It is found from Fig. 19 that increase in the ratio parameter $\lambda$ devaluates the fluid flow and decreases the induced velocity function $h(\eta)$. Fig. 20 shows the decreasing effect of $h(\eta)$ with increasing values of $M$. It is noticed from Fig. 21 that $h(\eta)$ increases by allowing the increasing values of injection, however, opposite effect are obtained from the increasing value of suction. Table 2 demonstrates the numerical results for velocity component $f^{\prime \prime}(0)$ for different values of We and $\lambda$ at $\alpha=M=f_{w}=0$ and auxiliary parameter $\hbar=-0.29$, which shows that the increasing values of $W e$ and $\lambda$ have positive impact on the velocity $f^{\prime \prime}(0)$. In contrast, the variation in the values of $M$ and $f_{w}$ provides the decreasing effect on velocity component $f^{\prime \prime}(0)$ as shown in Table 3 at $\alpha=W e=\lambda=0, \hbar=-0.29$. Additionally, the comparative analysis has been carried out for the velocity profiles $g(\eta)$, $h(\eta)$ and $k(\eta)$ in Figs. 28 - 30 with the Wang [9], which exhibits that the present results have excellent compatibility with the existing results.

\section{Closing remarks}

In the present paper the three dimensional non-aligned stagnation flow of second grade fluid over a porous rotating disk has been evaluated. HAM was used to solve the governing system of equations. The influences of var- 


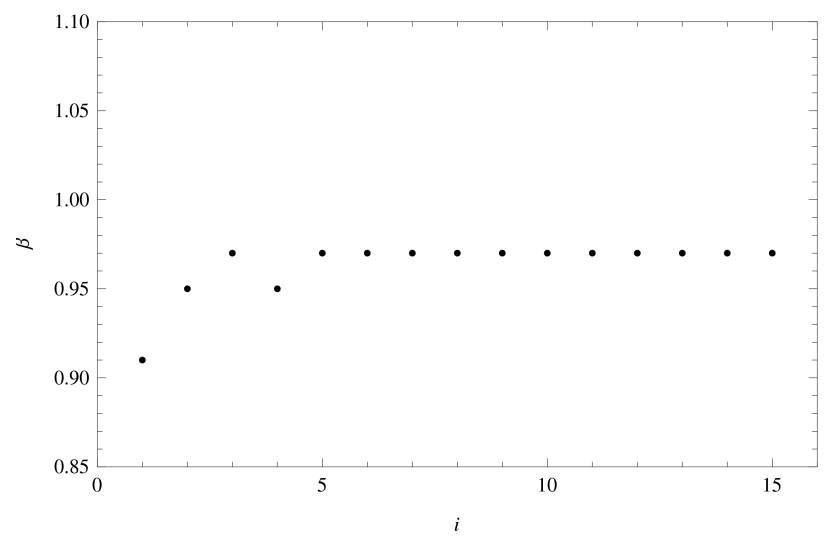

(a) $f$

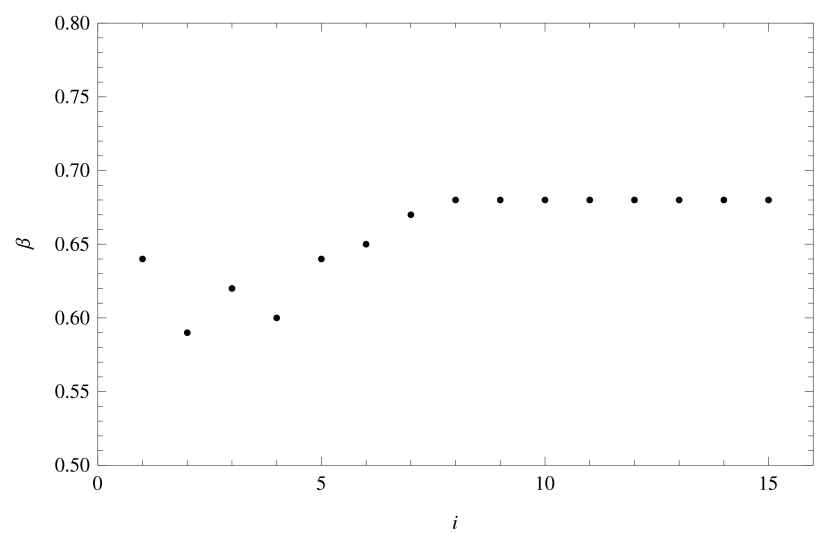

(c) $k$

Fig. 27: The ratio approach regarding to the presented solutions

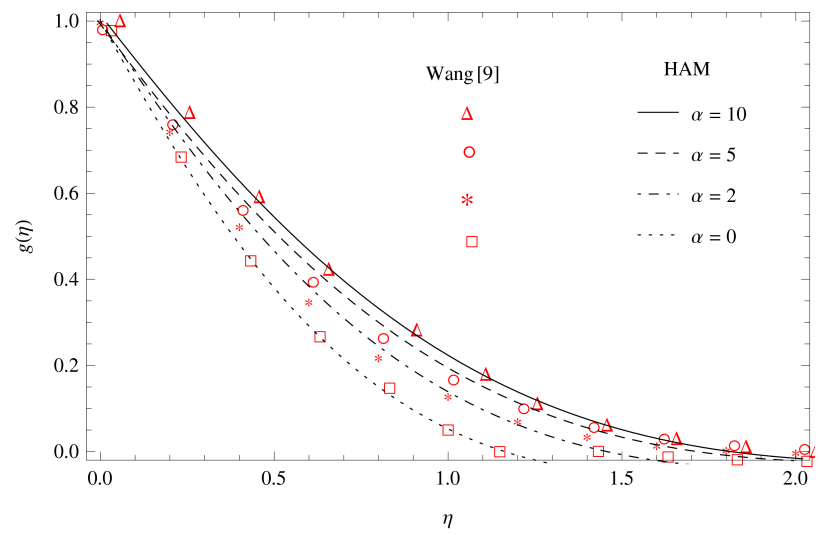

Fig. 28: Comparison of velocity profile $g$ for Wang [9] and HAM solution

ious parameters have been investigated on flow by plotting graphs. Numerical values of various velocity functions have been computed and compared results with Wang [9]. The outcomes of the study can be explained as:

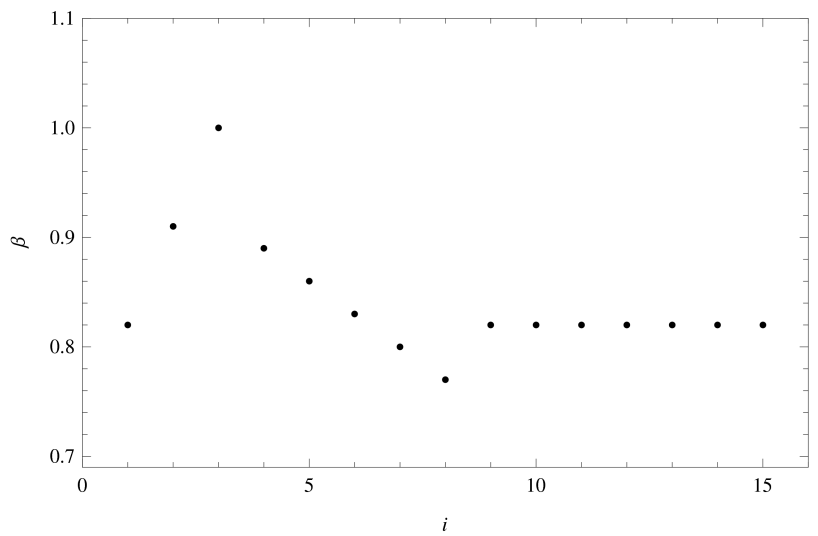

(b) $g$

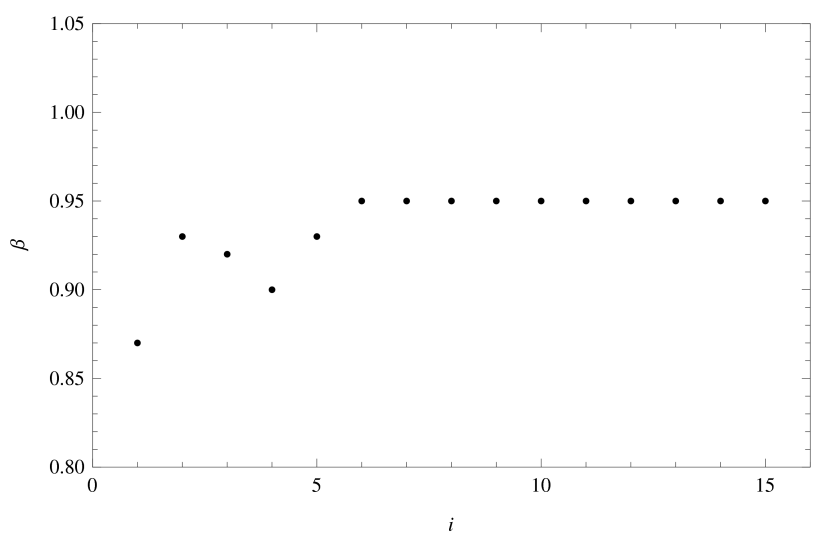

(d) $h$

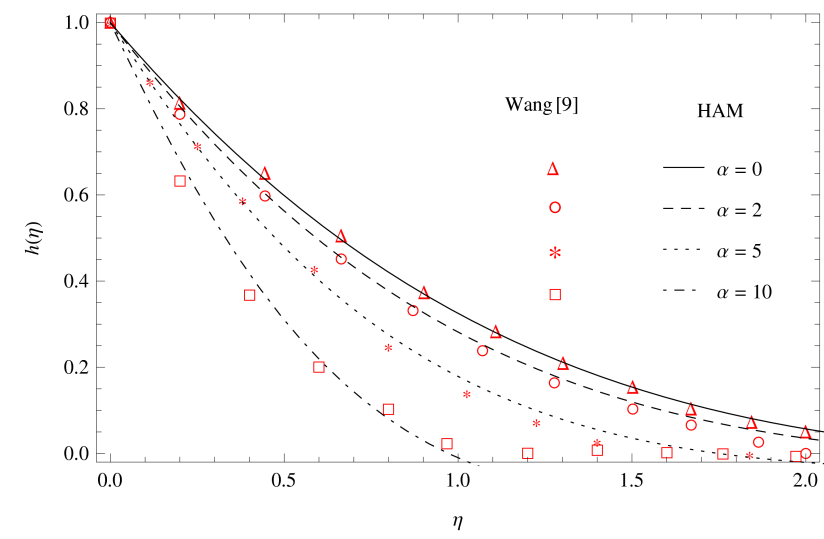

Fig. 29: Comparison of velocity profile $h$ for Wang [9] and HAM solution

- $\quad$ The velocity fields $f^{\prime}(\eta), g(\eta), k(\eta)$ increased by increasing $\alpha$ but the opposite relation was observed in $h(\eta)$.

- Increasing the values of We decreased $f^{\prime}(\eta)$ and $k(\eta)$ but increased $g(\eta)$ and $h(\eta)$. 


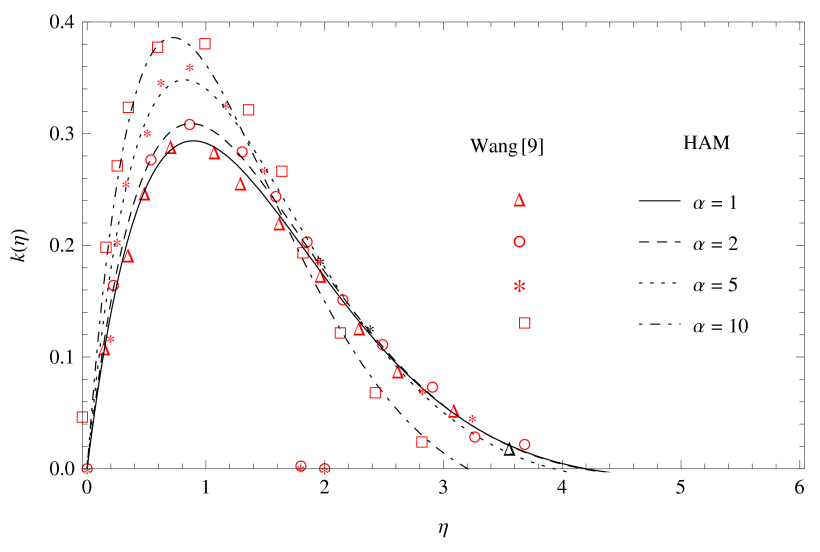

Fig. 30: Comparison of velocity profile $k$ for Wang [9] and HAM solution

- $\quad$ The effects of $\lambda$ and $M$ on $f^{\prime}(\eta), g(\eta)$ and $h(\eta)$ were quite similar.

- $\quad \lambda$ showed both increasing and decreasing effects on velocity component $k(\eta)$.

- $\quad g(\eta)$ and $k(\eta)$ decreased by allowing the increasing values of both suction $f_{w}>0$ and injection $f_{w}<0$.

- $\quad$ As the values of suction/injection increased, $f^{\prime}(\eta)$ also increased.

- Increasing the values of injection showed increasing effect on $h(\eta)$, whereas, the increasing value of suction exhibited decreasing effect on $h(\eta)$ and the higher values of $M$ decreased $k(\eta)$.

- The comparisons made good agreement of $f^{\prime}(\eta), g(\eta), k(\eta)$ and $h(\eta)$ with Wang [9] results.

Acknowledgement: The authors wish to express their sincere thanks to the esteemed reviewers for their attentive and thorough reading of this article and appreciate for their thoughtful comments and effective advice for the development of this manuscript.

\section{References}

[1] I. Ali, R.A. Shah, S. Islam, A. Khan, A.M. Siddiqui, Homotopy perturbation solution of second grade fluid through channels with porous walls of different permeability, World Appl. Sci. J., 8 (5) (2010) 536-542.

[2] A.M. Siddiqui, T. Haroon, Z. Bano, Slip effects on the flow of a second grade fluid in a varying width channel with application to stenosed artery,Uni. J. Appl. Math., 1 (2) (2013) 72-81.

[3] T. Hayat, S.A. Shehzad, M. Qasim, S. Obaidat, Flow of a second grade fluid with convective boundary conditions, Therm. Sci., 15 (2) (2011) 253-261.
[4] M. M. Rashidi, T. Hayat, T. Keimanesh, H. Yousefian, A study on heat transfer in a second-grade fluid through a porous medium with the modified differential transform method, Heat Transfer-Asian Res., 42 (1) (2013) 31-45.

[5] T. Von Kármán, Uber laminare und turbulente Reibung, ZAMM., 1(4) (1921) 233-252.

[6] T. Hayat, S. Iram, T. Javed, S. Asghar, Shrinking flow of second grade fluid in a rotating frame: An analytic solution, Commun. Nonlinear Sci. Numer. Simul. 15 (10) (2010) 2932-2941.

[7] M. Batista, Steady flow of incompressible fluid between two co-rotating disks, Appl. Math. Model., 35 (2011) 5225-5233.

[8] M. Turkyilmazoglu, Nanofluid flow and heat transfer due to a rotating disk, Comput. Fluids, 94 (2014) 139-146.

[9] C. Y. Wang, Off-centered stagnation flow towards a rotating disc, Int. J. Eng. Sci., 46 (2008) 391-396.

[10] S. Dinarvand, On explicit, purely analytic solutions of offcentered stagnation flow towards a rotating disc by means of HAM, Nonlinear Anal.; RWA 11(2010) 3389-3398.

[11] E. Erfani, M. M. Rashidi, A. B. Parsa, The modified differential transform method for solving off-centered stagnation flow toward a rotating disc, Int. J. Comput. Methods, 7 (2010) 655670.

[12] S. H. Nourbakhsh, A. A. Pasha Zanoosi, A. R. Shateri, Analytical solution for off-centered stagnation flow towards a rotating disc problem by homotopy analysis method with two auxiliary parameters, Commun. Nonlinear Sci. Numer. Simul., 16(7) (2011) 2772-2787.

[13] L. Bansal, Magnetofluiddynamics of viscous fluids, Jaipur Publishing House, Jaipur, India (1994).

[14] M. Turkyilmazoglu, Three dimensional MHD stagnation flow due to a stretchable rotating disk, Int. J. Heat Mass Tran., 55 (23-24) (2012) 6959-6965.

[15] M. Ashraf, A.R. Wehgal, MHD flow and heat transfer of micropolar fluid between two porous disks, Appl. Math. Mech. -Engl. Ed., 33 (1) (2012) 51-64.

[16] M. Ashraf, K. Batool, MHD flow and heat transfer of a micropolar fluid over a stretchable disk, J. Theor. Appl. Mech., 51 (1) (2013) 25-38.

[17] M. Turkyilmazoglu, Analytic approximate solutions of rotating disk boundary layer flow subject to a uniform suction or injection, Int. J. Mech. Sci., 52 (2010) 1735-1744.

[18] H.A. Attia, Rotating disk flow and heat transfer through a porous medium of a non-Newtonian fluid with suction and injection, Commun. Nonlinear Sci. Numer. Simul., 13 (2008) 1571-1580.

[19] J.E. Dunn, K.R. Rajagopal, Fluids of differential type-critical review and thermodynamic analysis, Int. J. Eng. Sci., 33 (1995) 689-729.

[20] K.R. Rajagopal, Flow of viscoelastic fluids between rotating discs, Theor. Compt. Fluid Dyn., 3 (1992 ) 185-206.

[21] R.L. Fosdick, K.R. Rajagopal, Anomalous features in the model of second order fluids, Arch. Ration. Mech. Anal., 70 (1979) 145-152.

[22] M. M. Rashidi, S. A. Mohimanian Pour, T. Hayat, S. Obaidat, Analytic approximate solutions for steady flow over a rotating disk in porous medium with heat transfer by homotopy analysis method, Comput. Fluids, 54 (2012) 1- 9.

[23] M. Turkyilmazoglu, An effective approach for evaluation of the optimal convergence control parameter in the homotopy analysis method, Filomat 30:6 (2016) 1633-1650. 
[24] M. Turkyilmazoglu, Numerical and analytical solutions for the flow and heat transfer near the equator of an MHD boundary layer over a porous rotating sphere, Int. J. Therm. Sci. 50 (2011) 831-842.

[25] S. J. Liao, Advances in the homotopy analysis method, World Scientific, 2014 